A N N A L E S Annales de Bretagne et des Pays de l'Ouest

\title{
Un feudiste de province au travail à la fin de
} l'Ancien Régime

A provincial feudiste at work at the end of the Ancien Régime

\section{Brigitte Maillard}

\section{(2) OpenEdition}

1 Journals

\section{Édition électronique}

URL : https://journals.openedition.org/abpo/4422

DOI : $10.4000 / a b p o .4422$

ISBN : 978-2-7535-7783-1

ISSN : $2108-6443$

\section{Éditeur}

Presses universitaires de Rennes

Édition imprimée

Date de publication : 20 mars 2019

Pagination : 127-161

ISBN : 978-2-7535-7782-4

ISSN : $0399-0826$

\section{Référence électronique}

Brigitte Maillard, «Un feudiste de province au travail à la fin de l'Ancien Régime », Annales de Bretagne et des Pays de l'Ouest [En ligne], 126-1 | 2019, mis en ligne le 01 janvier 2022, consulté le 07 janvier 2022. URL : http://journals.openedition.org/abpo/4422 ; DOI : https://doi.org/10.4000/abpo.4422 


\title{
Un feudiste de province au travail à la fin de l'Ancien Régime
}

\author{
Brigitte MAILLARD \\ Professeur émérite d'histoire moderne, université F. Rabelais - Tours
}

Dans la seconde moitié du XVIII ${ }^{\mathrm{e}}$ siècle, à partir de 1740 environ ${ }^{1}$, les parutions d'ouvrages traitant du droit féodalo-seigneurial se multiplient, qu'il s'agisse de traités rappelant les grands principes du droit, de dictionnaires ${ }^{2}$ à l'usage de juristes (officiers de justice royaux ou seigneuriaux, notaires) et même de seigneurs attentifs à la gestion de leurs terres ${ }^{3}$, ou de manuels destinés à des praticiens comme les feudistes ou commissaires à terrier ${ }^{4}$. Dans la vallée de la Loire, le principal auteur de tels ouvrages est un professeur de droit français à l'université d'Orléans, Pothier (1699-1772), qui jouit d'une renommée nationale et dont les traités parurent entre 1740 et 1781 . Tous ces ouvrages ont connu de nombreuses éditions, revues et augmentées; ils figuraient, entre autres, dans les bibliothèques des grandes

1. Par exemple : Bellami, Traité de la perfection, et confection des papiers terriers..., Paris, Paulus-du Mesnil et de Nully, 1746 ou Fréminville, Edme LA PoIX DE, La pratique universelle pour la rénovation des terriers et des droits seigneuriaux [...] dans lequel on trouvera tout ce qui est nécessaire pour bien faire la rénovation des censives et terriers, [...] le tout accompagné de modèles et stiles de tous les actes concernant cette matière, Paris, Morel, 5 vol., 1746-1757.

2. Ce type d'ouvrage est alors à la mode car d'usage pratique. Voir, par exemple, RENAUldon, Joseph, Dictionnaire des fiefs et des droits seigneuriaux, utiles et honorifiques, Paris, chez Delalain le jeune, $2^{\mathrm{e}}$ éd., 1788, p. 5. L'ouvrage est présenté comme "très utile et très commode à tous seigneurs, juges et avocats ".

3. "Les seigneurs même, qui lisent avec répugnance de longs traités de jurisprudence, pourront s'instruire de leurs droits et de leurs obligations en lisant attentivement la première partie " (Code des terriers ou principes sur les matières féodales, Paris, Prault et Vallat Lachapelle, 1771, p. v).

4. Voir BÉCHU, Philippe, "Le corpus de la littérature des feudistes. Problématique des auteurs et portée pratique des traités ", in BRUNEL, Ghislain, GUYOTJEANNIN, Olivier et alii, éd., Terriers et plans-terriers du XIII au XVIII siècle. Actes du colloque de Paris (sept. 1998), Rennes, PUR (BHR 5), 2002, p. 267-276. L'A. y étudie le contenu d'une petite partie (sept) de ces ouvrages. 
institutions religieuses ${ }^{5}$. Les manuels s'adressaient aux arpenteurs ${ }^{6}$ mais aussi aux " commissaires à terrier " ou feudistes, les spécialistes de la rénovation des terriers. Destinés à faciliter leur travail et à leur permettre d'améliorer grandement leurs méthodes pour réaliser des terriers " perpétuels ${ }^{7}$ ", ils avaient des buts pratiques et ne remettaient donc pas en cause les " traits féodaux " du droit de la propriété, alors que, dans leurs traités, certains spécialistes du droit français portaient un regard critique sur institutions féodales et privilèges et attaquaient la diversité régionale du droit coutumier ${ }^{8}$. La tâche des feudistes avait un but conservatoire; ils ne devaient pas apporter des modifications de fond dans les terriers qu'ils rénovaient, bien au contraire, mais dans la seconde moitié du XVIII ${ }^{\mathrm{e}}$ siècle, ils cherchèrent à en améliorer la forme, but qu'ils ont atteint, nous laissant de très beaux registres accompagnés de plans, parfois réunis en atlas, très réussis. Cette période semble avoir vu la rénovation de nombreux terriers $^{9}$, dans diverses régions. A-t-elle été un "âge d'or ${ }^{10}$ " des praticiens du droit féodal? Les rénovations de terrier sont-elles réellement devenues "légion ${ }^{11}$ "? Si oui, quel est leur sens puisqu'elles ne répondent pas à un besoin de remise en ordre utile après une période de troubles, comme ce fut le cas après les guerres de Religion? L'activité des feudistes reste encore mal connue. Une étude au plus près de leur travail dans les décennies qui précèdent l'été 1789, à partir de leurs archives malheureusement devenues rares, devrait permettre de mieux comprendre le rôle joué par les feudistes dans le maintien ou le développement de l'exercice des droits seigneuriaux.

5. Confisqués, ils ont constitué une partie des fonds des bibliothèques publiques nées sous la Révolution. Ainsi le fonds ancien de la médiathèque municipale d'Angers témoigne de la richesse en ce domaine des bibliothèques des grandes abbayes angevines, comme celles de Saint-Aubin ou Saint-Serge, même si au cours du XIX ${ }^{\mathrm{e}}$ siècle la bibliothèque publique créée en 1798 a perdu une partie importante de ses livres. Il est probable que ces ouvrages figuraient aussi dans la bibliothèque des seigneurs les plus importants.

6. DOYEN, Guillaume, Géométrie de l'arpenteur ou pratique de la géométrie en ce qui a rapport à l'arpentage, aux plans et cartes topographiques, avec une introduction à la rénovation des terriers, Paris, C. A. Jombert, 1769.

7. CLÉMENT DE BoISSY, Athanase, Le livre des seigneurs ou le papier terrier perpétuel qui indique la manière de renouveler les terriers et de les rendre utiles à perpétuité, pour la conservation des droits de la seigneurie, Paris, Cellot, 1776.

8. Renoux-Zagamé, Marie-Françoise, "Propriété ", in Bluche, François (dir.), Dictionnaire du Grand Siècle, Paris, Fayard, 1990, p. 1264.

9. BASTIER, Jean, La féodalité au siècle des Lumières dans la région de Toulouse (17301790), Paris, Bibliothèque nationale, 1975, p. 243. L'existence de ce grand mouvement de rénovation des terriers, qui aurait été engendré par une "réaction féodale ", selon l'expression de P. Sagnac, est souvent notée, mais l'état des archives seigneuriales en rend l'étude difficile.

10. SAINT-JACOB, Pierre de, Les paysans de la Bourgogne du Nord au dernier siècle de l'Ancien Régime, Paris, Les Belles-Lettres, 1960, p. 433.

11. "Les rénovations de terriers se multiplient dans la période 1750-1770, pour devenir légion au cours des deux dernières décennies de l'Ancien Régime " (AuBIN, Gérard, $L a$ Seigneurie en Bordelais d'après la pratique notariale (1715-1789), Publications de l'université de Rouen, 1989, p. 422). Toutefois, les historiens qui ont étudié cette question n'ont pu apporter aucune précision chiffrée à l'appui de cette affirmation. 
Les fonds anciens des Archives départementales de Maine-et-Loire conservent une partie des dossiers d'un feudiste qui exerça à Angers dans la seconde moitié du XVIII ${ }^{\mathrm{e}}$ siècle, Auguste Labry, qui eut sans doute un des plus gros cabinets de la ville, comme le montre la liste de ses clients. Son abondante correspondance ${ }^{12}$, active et passive, répartie entre 1752 et 1790 , a en grande partie ${ }^{13}$ trait à son activité professionnelle, en particulier pour la période 1780-1789, décennie essentielle dans l'histoire des seigneuries puisqu'elle apparaît a posteriori comme la fin de "l'Ancien Régime "; s'y ajoutent des documents concernant le fonctionnement du cabinet. Ce "fonds Labry " complété par d'autres archives, dont les titres féodaux de certains clients ${ }^{14}$, permet, malgré ses lacunes, l'étude de la clientèle, des méthodes et des pratiques du travail d'un feudiste dans les deux dernières décennies de l'Ancien Régime.

La zone d'observation est le centre de l'Anjou, province dans laquelle, au XVIII ${ }^{\mathrm{e}}$ siècle, le régime seigneurial reste vivant; à côté des grandes seigneuries, civiles comme le duché de Brissac ou ecclésiastiques telle la baronnie épiscopale de Chalonnes, il en subsiste une multitude de petites dont il est difficile de mesurer la vitalité ${ }^{15}$. Dans les années 1770 et 1780, l'Anjou, qui a retrouvé son statut d'apanage en 1771, ne semble pas connaître de grands mouvements anti-seigneuriaux; la seigneurie n'y apparaît pas comme agressive et les paysans ne sont pas désarmés face à elle. Dans les cahiers de doléances de 1789 , quels que soient leurs auteurs ${ }^{16}$, les plaintes contre les feudistes sont rares, - au contraire de celles, innombrables et graves,

12. Pourquoi ces dossiers, cotés de E 2961 à E 2985, ont-ils été conservés ? S'agit-il d'une confiscation révolutionnaire, Labry s'étant rangé dans le camp anti-révolutionnaire? On peut distinguer les archives professionnelles et les archives privées. Arch. dép. de Maine-et-Loire, E 2964-E 2971 : activités professionnelles (fonctionnement du cabinet). Id., E 2977-E 2985 (792 pièces) : correspondance passive, avec quelques réponses à l'état de brouillon. Id., E 2961-E 2963 et E 2972-2976 : affaires familiales. L'archiviste C. Port a classé ces lettres au XIX ${ }^{\mathrm{e}}$ siècle, par ordre alphabétique d'expéditeur; plusieurs sont sans date, leur en-tête ayant été découpé. Pour leur présentation détaillée, voir BÉcHu, Philippe, "Un feudiste et ses clients à la veille de la Révolution ", dans Plaisir d'archives. Recueil de travaux offerts à D. Neirinck, Mayenne, éd. de la Manufacture, 1997, p. 191-234.

13. La correspondance professionnelle est conservée à partir de 1770. Les lettres d'ordre privé et les papiers de famille donnent un aperçu de la vie familiale et sociale de Labry, qui dépasse le cadre angevin : son fils, clerc chez un négociant à Nantes, y a vécu les débuts de la Révolution; les courriers échangés avec ses trois frères installés à SaintDomingue évoquent la vie difficile de ces petits Blancs dans les Îles (Frostin, Charles, "Angevins de modeste condition établis à Saint-Domingue (correspondance Labry 17521778) ", Revue française d'Histoire d'Outre-Mer, t. LVII (1970), $\mathrm{n}^{\circ}$ 209, $4^{\mathrm{e}}$ trim., p. 447-468).

14. Le nom de Labry figure dans différents documents des séries E, G et H (archives du marquisat d'Étiau, des abbayes de Saint-Florent de Saumur, de Saint-Nicolas d'Angers, etc.); d'autres sont anonymes mais son intervention est connue par d'autres sources et son écriture, très soignée, est facilement reconnaissable.

15. Leur nombre est inconnu; beaucoup n'ont laissé que des bribes d'archives.

16. Le Moy, A., Cahiers de doléances des corporations de la ville d'Angers et des paroisses de la sénéchaussée particulière d'Angers pour les états généraux de 1789, 2 vol., Angers, Burdin et $\mathrm{C}^{\mathrm{ie}}, 1916$. 
contre les jurés-priseurs vendeurs de meubles ${ }^{17}$-, et ils ne semblent pas avoir subi de violences, ce qui ne fut pas le cas en Languedoc ou Velay ${ }^{18}$.

\section{Le feudiste et sa clientèle}

\section{La carrière de Labry}

Nous ne savons rien sur les études et la formation professionnelle de Labry, qui est né en décembre $1733^{19}$. Son milieu d'origine est étranger à la basoche, au moins du côté de son père qui était un médiocre marchand teinturier d'origine auvergnate. Par son mariage en $1766^{20}$, il entra dans la petite bourgeoisie : feu son beau-père, Péan Duchesnay, était marchand, ancien consul ${ }^{21}$. Son parent le plus important fut un notable angevin, Louis Michel Thorode, un cousin germain du côté maternel, " conseiller du roi, notaire honoraire, conseiller à l'hôtel de ville ", qui jouit d'une grande expérience comme notaire et "féodiste " et d'une solide réputation ${ }^{22}$. Labry n'a pas fréquenté la faculté des droits (sic) de l'université d'Angers, où le droit français était enseigné depuis la fin du XVII ${ }^{\mathrm{e}}$ siècle en application de l'édit de Saint-Germain (avril 1679) mais où, malgré la notoriété des Pocquet de Livonnière père ${ }^{23}$ et fils, n'exerça jamais un professeur de l'envergure de Pothier; il ne se dit jamais licencié ès-droit, avocat ou notaire, au contraire de nombreux autres feudistes ou commissaires à terrier ${ }^{24}$ à la fin du $\mathrm{XVIII}^{\mathrm{e}}$ siècle. Orphelin de père à 9 ans, il a sans doute fait son apprentissage

17. MARION, Marcel, Dictionnaire des institutions de la France aux XVII et XVIII siècles, Paris, Picard, 1923, p. 456. Les offices de " jurés priseurs vendeurs de meubles " créés en 1566, supprimés en 1771, ont été recréés et vendus à partir de 1780 . Sur chaque vente ils touchaient $4 \mathrm{~d}$ par livre de la vente et $3 \mathrm{l}$ par vacation de 3 heures, des droits de délivrance des rôles, sans compter leurs frais de déplacement, etc. Ils étaient accusés de faire monter les frais et d'absorber tout l'actif des petites successions.

18. NicOLAS, Jean, La rébellion française. Mouvements populaires et conscience sociale 1661-1789, Paris, Le Seuil, 2002, p. 185.

19. A. Labry est le dernier d'une fratrie de huit enfants, nés entre 1724 et 1733 . Un des sept garçons meurt vicaire à Longué le 15 avril 1761; les trois aînés partent entre 1751 et 1753 s'installer aux Îles.

20. Le mariage est célébré dans l'église Saint-Maurille le 19 août 1766. Il est suivi de trois naissances.

21. Le second époux de sa belle-sœur, veuve en premier mariage d'un médecin, était consul en exercice de la juridiction consulaire; grâce à sa démission, Labry reçut un brevet d'enseigne de la milice bourgeoise le 7 février 1766, peu avant son mariage (Arch. dép. de Maine-et-Loire, E 2961).

22. Notaire entre 1742 et 1759 , il a été ensuite secrétaire de l'église d'Angers dont il a trié et classé les papiers, puis receveur du censif du chapitre (Notice de la ville d'Angers par Thorode, LongIN, E. (dir.), Angers, Germain et Grassin, p. XI-XII). Sheridan, marquis d'Étiau, écrit en 1775 « Je suis fort reconnaissant aux bons conseils de M. Thorode. Je vous prie de lui faire des remerciements de ma part " (Arch. dép. de Maine-et-Loire, E 2915).

23. Claude Pocquet de Livonnière (1652-1726) y enseigna de 1689 à 1711. Il publia entre autres un Traité des fiefs (1729, 3 éditions) et les Règles du droit français (1730, 5 éditions); il donna aussi les Coutumes du pays et duché d'Anjou, conférées avec les Coutumes voisines [...] avec le Commentaire de M. Gabriel Dupineau (1725).

24. Les deux expressions semblent employées indifféremment; peut-être parle-t-on plutôt de commissaire à terrier quand le seigneur a obtenu des lettres à terrier. Le terme 
comme géomètre arpenteur ou comme clerc dans un cabinet de feudiste et/ou notaire, peut-être celui de son cousin ${ }^{25}$; il a dû y acquérir des savoirs très divers ${ }^{26}$. Il est qualifié de feudiste dès le 29 décembre 1756, dans l'acte d'inventaire après décès de sa mère, alors qu'il a juste 23 ans $^{27}$. Très jeune il démontre une excellente maîtrise de l'écriture : la qualité de sa signature, ornée d'un paraphe assez modeste, en atteste. Tout jeune homme un peu " frotté " au droit et aux " écritures anciennes ", comme Labry devait l'être, pouvait embrasser la profession de feudiste puisque pour celle-ci il n'existait pas de métier réglé ou juré ${ }^{28}$.

Jusqu'à la fin des années 1770, Labry a cumulé les fonctions d'officier seigneurial avec celles de feudiste : les actes de neuf nominations entre 1759 et 1779 ont été conservés ${ }^{29}$. Procureur fiscal dans plusieurs seigneuries $^{30}$, il a aussi été greffier de la baronnie de Sainte-Gemmes-sur-Loire en novembre 1766 et de la terre de Mûrs en octobre $1772^{31}$, puis celui de la baronnie de Champigné-le-Sec en 1776 et $1777^{32}$. Il a pu ainsi améliorer ses

" féodiste " ou " feudiste " aurait, pendant longtemps, signifié seulement " spécialiste des matières féodales".

25. C. Port le présente comme " héritier du feudiste Thorode " (PoRT, Célestin, Dictionnaire historique, géographique et biographique de Maine-et-Loire, Angers, Lachèse, 1876, t. II, p. 433-434).

26. Dans Le livre des seigneurs..., écrit en 1776, A. Clément de Boissy détaille en 183 pages de nombreux conseils pour les feudistes, fondés sur l'expérience du comté de Rostaing, en Blésois. Il insiste sur le soin, la minutie et l'attention dont le feudiste doit faire preuve.

27. Arch. dép. de Maine-et-Loire, E 2961. Il a sans doute été arpenteur à ses débuts. Dans l'inventaire dressé lors de son mariage le 18 août 1766 (Arch. dép. de Maine-et-Loire, E 2961. Minutes Bardoul), figurent des instruments nécessaires pour lever des plans : un " graphomètre ", une boussole, un " étui de mathématique ", une chaîne, dix fiches de fer, une lunette d'approche (valeur totale : 60 lt), ainsi que 12 feuilles de grand papier à dessin (valeur 12 lt), 2 rames de grand papier (50 lt). Sa bibliothèque comporte " centvingt volumes de livres tant de dévotion que d'histoire et pratique ", malheureusement non détaillés. Il a déjà travaillé pour des seigneurs : dans ses dettes actives, il déclare qu'il lui est " dû par différents seigneurs tant pour avances que pour travail que [il a] fait pour leurs fiefs » $1800 \mathrm{lt}$, soit $22 \%$ de l'actif total (8196 lt).

28. Labry ne fut jamais officier du roi mais s'intégra dans la bourgeoisie angevine. Il devient officier de la milice en 1766 et il est reçu franc-maçon en 1770, à 37 ans : il s'adresse à ses " frères " quand il a besoin d'argent. Le 28 août 1785 , ne pouvant se faire payer par ses clients, il demande à Blanchard de Pégon de lui prêter " une pierre plate pesant 600 lt " jusqu'à la Saint-Martin mais en mai 1787, il n'a toujours rien remboursé (Arch. dép. de Maine-et-Loire, E 2977). Il devient aussi propriétaire foncier : il acquiert différents biens (immeubles et terres); il est donc lui-même pris dans le système seigneurial. En avril 1786, il crée, avec ses fils, des parents et son associé Aubin, une société pour accenser des landes dans la paroisse de Briollay (Id., E 2961).

29. Arch. dép. de Maine-et-Loire, E 2964. Le 2 octobre 1759, Thorode, sénéchal du fief de Monnet (à Beaufort), reçoit son cousin Labry comme procureur fiscal.

30. Labry est nommé procureur fiscal des terres de la Bardouillière etc., à Cuon, le 10 juillet 1761, puis à nouveau le 8 juin 1765; la désignation vaudrait donc pour 4 ans.

31. Ces terres appartenaient à la famille du trésorier de la Marine Baudard de SaintJames (ou Sainte-Gemmes).

32. Arch. dép. de Maine-et-Loire, H 241. La terre (paroisses de Souzay, Chacé...) relève de l'abbaye Saint-Aubin d'Angers. Aux assises de 1776-1777, Labry est greffier et il n'est pas l'auteur du terrier réalisé ultérieurement. 
revenus et acquérir une connaissance approfondie du fonctionnement des seigneuries. Il a aussi agi comme « fondé de pouvoir " de quelques seigneurs importants, comme le marquis d'Harcourt, retenu à Paris pour le service du roi $^{33}$. Il jouit d'une bonne réputation : en 1779, son associé Aubin se plaint que, s'il est seul pour tenir des assises, " on demande monsieur Labry et [si] on dit qu'il n'y est pas, il y en a qui forment des difficultés de rendre ne le voyant pas $^{34}$ ".

\section{La clientèle de Labry}

Pour se constituer une clientèle la concurrence est certainement rude ${ }^{35}$ : disposer d'un bon réseau de relations est essentiel. Labry peut sans doute compter sur son cousin Thorode et même sur certains clients. Ainsi en mars 1770, La Bardouillère lui écrit être prêt à dire " avec un grand plaisir tout ce qu'il faut dire en cas que l'on me parle de la part de M. de Sheridan et je désire bien sincèrement que vous vous arrangiez avec lui ${ }^{36}$ ". D'autres lui signalent des clients potentiels : le $1^{\mathrm{er}}$ septembre $1787^{37} \mathrm{il}$ apprend que " les feudistes n'ont point encore passé " à Bierné pour les fiefs de la Barre, propriétés d'un Lavallois, Dubois, contrôleur des guerres, qui, " riche d'environ un million ", vient d'acquérir dans le Maine une terre de plus de $500000 \mathrm{lt}$; on lui conseille de faire intervenir en sa faveur Boisjourdan, un de ses clients, et le curé de la paroisse, un Angevin... Des liens de parenté existent entre certains clients de Labry, qu'ils ont pu se recommander mutuellement : Maillé de La Tour-Landry, seigneur d'Étiau, de Jalesnes..., a des relations familiales et amicales avec la famille Le Jumeau, baron de $\mathrm{Blou}^{38}$.

Les lettres professionnelles conservées émanent de nombreux correspondants; souvent isolées et donc peu utilisables, elles révèlent néanmoins

33. Arch. dép. de Maine-et-Loire, E 2978. Il va rendre foi et hommage au marquisat de Beaupréau pour la seigneurie de Vieil-Baugé, au nom de Charles de Harcourt qui a acquis cette terre en juin 1764, grâce à un retrait lignager au nom de sa femme. Son conseil, réuni le 11 février 1781, jugea que le projet de foi et hommage risquait d'être trouvé très insuffisant et donc d'être impuni; il ordonna alors à Labry de renouveler sa démarche.

34. Arch. dép. de Maine-et-Loire, E 2968.

35. Dans les Affiches d'Angers, journal bimensuel qui paraît à partir de 1773, de nombreux individus font des offres aux seigneurs. Écrit-il à différents clients pour proposer ses services comme le faisait G. Babeuf? Il ne reste pas trace de telles démarches (PELLETIER, Antoine, "Babeuf, feudiste ", Annales historiques de la Révolution française, 1965, n 179, p. 35).

36. Arch. dép. de Maine-et-Loire, E 2978. Sheridan a effectivement chargé Labry de la rénovation du terrier pour le marquisat d'Étiau, acheté en 1767. Toutefois, dans le " registre des dépenses domestiques " de Jalesnes entre 1769 et 1784, aucune dépense concernant le terrier n'est notée (Id., 1E 395).

37. Arch. dép. de Maine-et-Loire, E 2970.

38. Lorsque Charles Henry François, comte de Maillé de La Tour-Landry, épouse Jeanne Sheridan, dame d'Étiau, son témoin est René Toussaint Le Jumeau, baron de Blou, qui est aussi son beau-frère (Arch. dép. de Maine-et-Loire, RP Saint-Philbert-du-Peuple, 5 décembre 1780). 
l'importance de la clientèle de Labry. Les demandes sont de deux types. Très souvent il s'agit de tâches limitées relevant d'un spécialiste du droit seigneurial. Ainsi, entre 1775 et 1778 , pour un groupe de huit clients Labry travaille vingt-huit jours et ses clercs soixante-quatorze ${ }^{39}$. Le 16 décembre 1782, Pays du Vau, seigneur de Lathan, soucieux de mieux gérer ses landes lui demande " un service, le tout à [ses] moments perdus ", c'est " d'arpenter toutes [ses] landes et en faire un plan figuré [...]. Cette besogne demande un feudiste habile et intègre, voilà pourquoi je m'adresse à vous de préférence ${ }^{40}$ " sur les conseils de Thorode. Il peut aussi être sollicité pour la rédaction d'un aveu ${ }^{41}$ ou pour la copie d'un acte ${ }^{42}$. Assez souvent, il lui est demandé de régler un litige entre seigneurs ${ }^{43}$. Les commandes les plus importantes, et les plus lucratives, concernent la rénovation d'un papier terrier ${ }^{44}$. Pour les grandes seigneuries, un contrat est alors passé par écrit, souvent sous seing privé ${ }^{45}$; sinon, il reste tacite : en mars 1779 , Labry déclare s'être " arrangé " avec le prieur de Cheviré-le-Rouge.

\section{Les clients laïques}

Les seigneurs laïques, hommes ou femmes, sont nombreux, comme le montre la liste suivante, qui n'est pas exhaustive ${ }^{46}$ :

- 15 décembre 1770 : Jacques Sheridan pour le marquisat d'Étiau ${ }^{47}$,

- septembre 1771 : madame de Millon pour le fief de la Poissonnière en Combres (Saint-Clément-des-Levées),

39. Arch. dép. de Maine-et-Loire, E 2967. "Semainier où sont portées les journées de MM. mes clercs et pour qui ils les ont employés " (1775-1787). Ce registre (38 feuillets) est complet et constitue donc une source fiable.

40. Arch. dép. de Maine-et-Loire, E 2984. Labry accepta et confia le travail à Ancenay, ingénieur géographe du roi et inspecteur des travaux de l'Authion.

41. Le 11 octobre 1781, madame Loüet de Cordais demande à Labry de rédiger l'aveu qu'elle doit rendre au baron de Blou : "puisque la chause presse, nous vous prions de voulouer bien vous transporter icy le plus tos possible pour travailler à notre aveu " (Arch. dép. de Maine-et-Loire, E 2982). Toussaint Henry Le Jumeau, qui hérite en 1785 des terres de Blou, lui demande en 1786-1787 l'aveu des châtellenies de Neuillé et Vivy à rendre « à haute et puissante dame Agnès O'Chiel, comtesse de Trèves, veuve de haut et puissant seigneur Jean Stapleton, chevalier, comte de Trêves, seigneur de Cunault, Pocé et autres lieux "; le procureur fiscal de Trêves ayant critiqué le projet, il fallut une " addition et réformation" (Id., E 166).

42. Arch. dép. de Maine-et-Loire, E 2982. Lenée notaire à Allones demande copie de la déclaration féodale faite au fief de Blou " le plus promptement possible " (27 avril 1786).

43. Cf. Arch. dép. de Maine-et-Loire, E 2977, E 2983, E 2984, E 2997.

44. Pour ces clients aussi Labry joue le rôle de conseiller en matière féodale. Le 14 novembre 1785, Boisjourdan lui écrit : «J'avais besoin de conseils que vous m'eussiez donné, Monsieur, si vous fussiez venu comme je l'espérais. Je suis attaqué de toutes parts par les feudistes" (Arch. dép. de Maine-et-Loire, E 2977).

45. Comme dans la région de Toulouse, mais au contraire du Bordelais, les contrats notariés sont rares (BASTIER, Jean, La féodalité au siècle des Lumières..., op. cit., p. 244).

46. Les contrats conservés sont presque tous dans la liasse E 2971/1 et 2 mais aucun n'est antérieur à 1770; d'autres ont disparu mais les dates en sont connues.

47. Arch. dép. de Maine-et-Loire, E 542. 
- mars 1773 : Talour de la Cartrie pour le fief de la Gouberie (VieilBaugé) ${ }^{48}$,

- janvier 1774 : madame de la Gasnerie, pour le fief de Fougeré (FontaineMilon),

- 14 juin 1774 : du Lis, pour le fief de la Motte de Croustelle en Poitou,

- $1^{\text {er }}$ juillet 1776 : « marché fait verbalement » avec le baron de Blou ${ }^{49}$,

- février 1778 : madame de Gironde pour Montfaucon (dans le Maine),

- octobre 1778? : le marquis d'Harcourt pour la châtellenie du Plessisau-Jau (Mazé) ${ }^{50}$,

- août 1779 : le marquis de Maillé pour les terres de Jalesnes, PlessisBatard, le Pin, les Forêts (Vernantes) ${ }^{51}$,

- 26 juin 1780 : Jeanne de Sheridan, pour l'achèvement du terrier du marquisat d'Étiau ${ }^{52}$, hérité de son père,

- printemps 1782 : concession, par le conseil de Monsieur frère du roi, de la réalisation du terrier des " fiefs et seigneuries dépendant du château et domaine de Baugé " à " la société " conclue en février 1782, par Bretonneau, receveur des domaines de Monsieur à Baugé avec Labry et Aubin ${ }^{53}$,

48. Arch. dép. de Maine-et-Loire, E 2969. Labry est payé 6 lt par jour quand il travaille " en ville " et 8 lt quand il est " en campagne ». En 1774, il fait tenir les assises et encaisse 155117 s 4 d.

49. Située près de Longué, à l'est d'Angers, la baronnie de Blou appartient alors depuis plus d'un siècle à la famille Le Jumeau. René Toussaint, baron de Blou, etc. est un client important : entre juillet 1776 et juin 1780, Labry lui a consacré 35,5 jours de travail à 6 lt par jour et surtout ses clercs 1053,5 jours, à 4 lt par personne, pour un coût total de 4395 lt (Arch. dép. de Maine-et-Loire, E 2969). En 1771, le feudiste Pierre Desportes avait été chargé du classement des titres de la baronnie de Ramefort, à Blou, arrentée de l'évêque d'Angers (Id., G 192).

50. Terre membre de la baronnie de Fontaine-Guérin, propriété à la fin du XVIII ${ }^{\mathrm{e}}$ siècle des Harcourt. Entre octobre 1778 et juin 1780, Labry travailla 341 jours et ses clercs 753,5 jours, pour un coût de 5122 lt et des frais de transport de 117 lt (location de chevaux). En mai 1779, Labry reçoit 2000 lt et en janvier 1780, 1000 lt. de Charles Louis Hector seigneur depuis 1774.

51. Arch. dép. de Maine-et-Loire, E 2968. Le censif prévu n'est pas réalisé car, après la mise en ordre des titres et la réalisation des tables, le marquis déclare ne pas en vouloir et fait cesser le travail. Toujours impécunieux, il a demandé à Labry de rechercher " quelques aubaines de fief".

52. Labry doit aussi finir sous un an le terrier des autres fiefs selon le contrat passé avec Sheridan. Le 5 décembre suivant, le marquis de Maillé La Tour-Landry épouse, à Saint-Philbert-du-Peuple, Jeanne Sheridan, marquise d'Étiau, fille et héritière de Jacques Sheridan († 19 avril 1780). Après 1781, le projet d'un censif pour le fief d'Étiau est établi non par Labry mais par Desportes et Maillard, feudistes associés (Arch. dép. de Maineet-Loire, 1E 278).

53. Arch. dép. de Maine-et-Loire, E 2968. Ce contrat a été un des plus importants du cabinet de Labry. En 1771, le domaine de Baugé était une des «parties [du] Domaine qui ont été engagées "; le duc d'Estissac fut l'engagiste jusqu'en 1779. Le 22 février 1777, son conseil représente à Monsieur « que la confusion et le désordre qui règnent dans les mouvances de son apanage, les usurpations sans nombre qui ont été faites sur le Domaine, 
- 2 septembre 1783 : Louis François Séraphin de Boisjourdan, seigneur de Longuefuye, les Courants, pour la terre des Courants, châtellenie de Longuefuye et annexes, au Maine,

- 5 novembre 1783 : Charles Jean-Baptiste Morel de La Motte, seigneur de la Motte, des Landelis [Landelles], Lépinay et autres lieux, pour la terre de Lépinay (Combrée) ${ }^{54}$,

- 18 février 1784 : Charles François de Maillé La Tour-Landry, marquis de Jalesnes, et Charles Henry, comte de Maillé La Tour-Landry pour les fiefs de Jalesnes ${ }^{55}$, du Plessix-Botard, du Pin, de la Papottière, etc.,

- 15 décembre 1784 : Marie-Joseph Charles Tessier de Bellère pour les fiefs dépendant de la Roche-Noyau (Vernoil-le-Fourrier).

Il faut ajouter les clients dont les contrats sont antérieurs à 1770 ou postérieurs à 1784, dont il ne subsiste pas de trace écrite, tel celui conclu pour les terriers des fiefs constituant le comté de Sainte-Gemmes-d'Andigné : Labry, qui est témoin lors des assises de 1784 et 1785, en a été, au moins pour partie, l'auteur ${ }^{56}$. En mai 1785, parmi ses clients figurent de grandes familles de la noblesse angevine, comme Constantin de La Lorie, le comte d'Andigné, madame de La Devansais. Cette clientèle est en très grande majorité noble ${ }^{57}$.

Si une recrudescence de l'activité des feudistes, difficile à mesurer, se produit à la fin du XVIII ${ }^{\mathrm{e}}$ siècle en Anjou, elle peut être la conséquence des transferts de propriété au sein de la noblesse, bien constatés ailleurs en France : des officiers du roi ou de nouveaux nobles ${ }^{58}$ acquièrent des seigneuries et, souhaitant mettre de l'ordre dans leurs titres pour affirmer leur nouveau statut social et connaître exactement l'étendue de leurs droits afin de toucher leurs revenus, ont recours aux feudistes pour mettre à jour leurs terriers; ils sont prêts à consentir l'effort financier nécessaire. Ainsi Sheridan, un jacobite irlandais établi à Nantes ${ }^{59}$, acquiert en juillet 1767 le marquisat d'Étiau, la baronnie d'Avoir, la châtellenie de Longué et il passe,

exigeraient qu'il soit procédé incessamment à la confection d'un terrier général comme le seul moyen de rétablir le domaine dans son intégrité " (Arch. nat., R/5/145.)

54. Arch. dép. de Maine-et-Loire, E 2971/2.

55. Arch. dép. de Maine-et-Loire, E 2971/2. En décembre 1783, le contrat précédemment passé par convention orale entre Maillé et Labry a été dénoncé, pour des raisons financières, dans un accord conclu sous seing privé.

56. Arch. dép. de Maine-et-Loire, E 1265, E 1266, E 1273. Les registres ne sont pas signés, mais leur écriture est identique à celle de Labry.

57. Il est difficile de connaître le statut social exact de certains clients. Laurent Bourquin dénombre pour l'Anjou en 1789433 familles nobles, établies en majorité dans les élections d'Angers et de Saumur, zones où Labry recrute ses clients, et qui ont connu au XVIII ${ }^{\mathrm{e}}$ siècle un fort renouvellement des lignages ("Les mutations du peuplement nobiliaire angevin à l'époque moderne ", Histoire, Economie \& Société, 17/2, 1998, p. 241-259).

58. Toutefois, les principaux clients laïques de Labry ne sont pas des officiers du roi ou de tout nouveaux venus dans la noblesse, à la notable exception de Sheridan.

59. On peut noter que les lettres de " reconnaissance d'ancienneté " de noblesse pour les trois frères Sheridan, jacobites irlandais résidant à Nantes, ont été signées l'année précédente, en juin 1766 (Arch. nat., O/1/110). 
en 1770, un contrat avec Labry, dont ce fut un des plus gros chantiers : avec ses clercs il y travailla 4764 jours, pour des honoraires de 20460 lt. entre décembre 1770 et avril 1780, date du décès de Sheridan, alors même que le travail n'était pas encore achevé.

\section{Les clients ecclésiastiques}

À l'instar des laïcs, des institutions ecclésiastiques sont des clients épisodiques. En 1770, Labry travaille deux jours pour l'abbaye Saint-Serge d'Angers, en 1779-1780, quatorze jours et ses clercs onze jours et demi pour le chapitre cathédral ${ }^{60}$. Après 1780 , il s'occupe aussi des fiefs du chapitre Saint-Pierre d'Angers. Parmi les rares séculiers qui s'adressent à lui, il y a surtout les prieurs successifs de Cheviré-le-Rouge. La Motte, curé de Sarrigné, passe un accord avec lui le 24 mars 1774; entre cette date et septembre 1776 Labry lui consacre cinquante-cinq jours et ses clercs trois cent quatre-vingt-dix, pour un coût total de $2190 \mathrm{lt}$; il finit le travail sous les deux prieurs suivants.

Trois grandes abbayes angevines sont de gros clients : celles de SaintMaur (du 2 mars 1770 à juillet 1776), de Saint-Nicolas ${ }^{61}$ à Angers (de février 1771 à juin 1776), et l'abbaye royale de Saint-Florent près Saumur, pour les fiefs de Nantilly et Varrains (de juin 1775 à mai 1780) ${ }^{62}$. Elles recherchent une mise en ordre de leurs terriers pour un meilleur rendement financier.

60. Thorode a été le feudiste du chapitre cathédral. Le travail de remise en ordre du chartrier, prévu par un contrat du 14 juin 1760, a nécessité 1767 jours de travail et a coûté, y compris les frais, 13754 lt (Arch. dép. de Maine-et-Loire, G 270). En 1770, Labry prend peut-être en partie sa succession, après l'achèvement du " Répertoire général [...] des titres du chapitre " (Id., G 270); il réalise le papier terrier de la terre d'Aubigné-Briand et Vocalé, malheureusement non daté (Id., G 433). Le chapitre cathédral fait aussi beaucoup travailler le feudiste Jean-Gabriel Cloquet.

61. Labry a plusieurs concurrents, dont Mathurin Richou, notaire royal, un des feudistes les plus actifs de la place à la fin du XVIII ${ }^{\mathrm{e}}$ siècle. L'abbaye de Saint-Florent l'emploie : en tant que «commissaire nommé pour la confection dud. terrier par sentence de Monsieur le lieutenant général de Saumur ", il signe un traité pour rédiger un " censif modernisé " du domaine de Verrie (Arch. dép. de Maine-et-Loire, H 2442, H 2443 et H 2444); il réalise en 1778-1780 les terriers des fiefs tenus dans les paroisses des Ulmes (Id., H 2247-2248), Meigné et Verrie (Id., H 2229) et il participe à leurs assises (Id.). En 17831784, il réalise le censif du prieuré Saint-Pierre de Montreuil-Bellay pour l'abbaye SaintNicolas (Id., H 687), tandis que, en 1783, René Dezé Maisonneuve renouvelle le papier terrier de la terre de la Touche à l'Abbé, à Sainte-Gemmes-d'Andigné (Id., H 712) et qu'en 1785, Guillou confectionne le censif de la Cellererie (Id., H 538).

62. Arch. dép. de Maine-et-Loire, H 2812. 


\section{Temps de travail du cabinet de Labry et coût pour les abbayes ange-} vines (entre 1770 et 1780)

\begin{tabular}{|l|c|c|c|c|c|c|}
\hline \multicolumn{1}{|c|}{ Abbaye } & \multicolumn{2}{|c|}{ Travail de Labry } & \multicolumn{2}{c|}{ Travail des clercs } & \multicolumn{2}{c|}{ Total } \\
\hline & en jours & en lt & en jours & en lt & en jours & en lt \\
\hline Saint-Nicolas & 193,5 & 1162 & 433,5 & 1738 & 627 & 2900 \\
\hline Saint-Florent & 100,5 & 603 & 676 & 2704 & 776,5 & 3307 \\
\hline Saint-Maur & 124 & 744 & 2499 & 9996 & 2623 & 10740 \\
\hline Total & 418 & 2509 & 3608,5 & 14438 & 4026,5 & 16947 \\
\hline
\end{tabular}

Entre 1771 et $1779^{63}$ Labry comptabilise pour lui-même 2053 jours de travail, dont 81,7 \% pour les laïcs, et pour ses clercs 11697 jours, dont $68 \%$ pour les laïcs. Le cabinet a donc consacré presque un tiers (30,6\%) de son activité à la féodalité de seigneurs ecclésiastiques, preuve de la puissance de l'Église angevine ${ }^{64}$. Ses clients résident à proximité d'Angers, plutôt à l'est, en particulier dans la vallée de la Loire, zone la plus riche de l'Anjou, et au nord de la province; ils sont le plus souvent à moins de 50 km, distance que le feudiste peut parcourir aisément. Deux clients notables résident en dehors de l'Anjou : Boisjourdan, seigneur de Longuefuye au Maine, et un Angevin établi en Poitou ${ }^{65}$.

Labry doit faire fonctionner son cabinet et il ne néglige aucun client. Les vastes chantiers sont évidemment financièrement les plus intéressants mais ils exigent beaucoup de temps et in fine, Labry ne peut travailler que pour un tout petit nombre de seigneurs : durant l'été 1778, ses clercs se consacrent au marquisat d'Étiau; au printemps de 1781, à quatre clients, dont le principal est le baron de Blou (90 jours sur 123,5); après 1782, au domaine de Baugé. La chronologie des contrats montre que, sauf si les lacunes de la documentation sont très importantes, Labry ne signe pas plus de deux ou trois gros contrats par an; ses collègues ne font certainement pas autrement. Même pour une petite seigneurie le travail de confection d'un terrier occupe, pendant des mois, parfois des années, plusieurs personnes à Angers comme en campagne.

63. Arch. dép. de Maine-et-Loire, E 2969.

64. Selon Georges Lefebvre, dans le Nord de nombreuses institutions ecclésiastiques ont aussi fait refaire leurs terriers à ce moment (Les Paysans du Nord pendant la Révolution française, Paris, Colin, p. 150).

65. Michel Laurent de Falloux, fils du lieutenant général en la sénéchaussée d'Anjou, a épousé en septembre 1773 l'héritière du fief de la Mothe de Croustelle, Marie-Suzanne Poussineau de la Mothe (PoRT, Célestin, Dictionnaire..., op. cit., t. II, p. 133). Labry avait commencé à travailler en juin 1774 au terrier de cette terre (39,5 jours et 313 jours de clercs (dont une partie sur place), pour un coût de 1176 lt, plus les frais) (Arch. dép. de Maine-et-Loire, E 2969). Le 23 août 1777, suite à un litige avec le chapelain, abbé commendataire de Fontenay-le-Comte, Falloux est reconnu seigneur haut justicier du bourg (Arch. dép. de la Vienne, 1H15). C'est sans doute ce conflit qui l'a amené à demander, pour faire rétablir ses droits, la remise en ordre du chartrier à un feudiste qu'il connaissait, bien que les déplacements fussent longs. 


\section{Le fonctionnement du cabinet}

Labry a été un employeur important. Ses clercs sont des salariés à temps plein, embauchés avec un contrat de travail; selon les besoins, il recrute en campagne divers individus pour un temps limité. Ces hommes doivent présenter des qualités professionnelles : avoir une "belle écriture " et connaître la féodalité ${ }^{66}$ pour les clercs et les " écrivains ", savoir lever des plans pour les géomètres. Dans une annonce des Affiches d'Angers, le 30 janvier 1784, un feudiste recherche " deux ou trois jeunes gens sages et raisonnables qui écriraient supérieurement et qui auraient travaillé plusieurs années à la féodalité "; dans une autre, du 19 novembre 1784, " le commissaire à terrier du duché de Thouars " indique qu'il " désirerait trouver plusieurs jeunes gens instruits dans la partie des plans et sachant déchiffrer les vieux titres et les mettre en belle écriture moderne ". Des talents paléographiques sont nécessaires car, loin de les négliger et au pire de s'en débarrasser, les clercs doivent pouvoir déchiffrer pour les copier les titres les plus anciens qui ont une grande valeur.

\section{Les collaborateurs occasionnels}

Auxiliaires des clercs, les "écrivains " sont chargés en campagne d'écrire des actes, de préparer les convocations aux assises et/ou de faire des copies. Pour le gros chantier du terrier de Baugé, à partir d'avril 1783, il faut recruter sur place plusieurs hommes ${ }^{67}$. Le dénommé Ratouis, habitant Vernoil, propose ses services à Labry; il jouit de la recommandation de Bretonneau qui parle de sa " très belle écriture " et de sa bonne connaissance du pays... mais qui signale aussi qu'il " aime un peu trop le vin "; il coûterait 300 à 400 lt par an, non logé, non nourri. À l'été 1784, pour le remplacer car il est " toujours en ribote", le même Bretonneau propose " un travailleur [...] qui écrit fort bien et propose de faire tout ce qu'il y aura à faire à tant la feuille. Il est marié et par conséquent sera sédentaire, et je crois qu'il convient très bien ".

Les arpenteurs géomètres ont un rôle grandissant. Selon Fréminville, le feudiste doit " lever les plans des lieux lui-même, à moins qu'il n'ait un géomètre dont il connaisse parfaitement la capacité ${ }^{68}$ ". Les arpenteurs ${ }^{69}$ doivent savoir au moins " travailler avec la planchette ". Labry leur fournit différents instruments, comme des " chaînes d'arpenteur " dont certaines font 22 pieds ou une «boussole à genoux pour les chemins et les sinuosités des rivières " (en 1770 il en fait acheter à Paris pour 60 lt). Ils doivent aussi

66. Arch. dép. de Maine-et-Loire, E 2378, lettre de Trottier à Labry, $1^{\mathrm{er}}$ novembre 1784 .

67. Arch. dép. de Maine-et-Loire, E 2985 et E 2978.

68. FrÉminVILLE, Edme LA PoIX DE, Les vrais principes..., Paris, Valleyre père, 1764, t. II, p. 294.

69. On ignore comment Labry les recrute et combien il les rémunère. Dans le Blésois, le salaire des arpenteurs est de 10 sous par arpent; il est moins élevé dans « les pays où le sol n'est pas autant divisé " (Le livre des seigneurs..., p. 69). Selon Fréminville, la taxe d'arpentage ne peut dépasser 20 sous/arpent. 
maîtriser la diversité des unités de mesure. Dans le projet d'aveu pour une terre relevant de la châtellenie de Fontaine-Milon ${ }^{70}$, de nombreux articles " paraissent justes pour les quantités et confrontations, en les comptant à raison de 10 perches par boisselée, mais si au contraire on ne veut les employer qu'à raison de 8 perches un tiers de perche par boisselée, on les rectifiera dans le corps dud. projet d'aveu ". Les "indicateurs requis pour la reconnaissance des bornes et limites " qui les accompagnent sur place leur permettent un repérage exact des parcelles; choisis parmi les habitants du lieu jugés honnêtes et connaissant très bien le terrain, ils sont payés par Labry.

Le travail en extérieur est parfois pénible : le 16 janvier 1782, Ancenay, ingénieur géographe du roi et inspecteur des travaux de l'Authion, arpenteur juré pour le comté de Beaufort, a mis plus de huit jours pour réaliser l'arpentage des marais du Lathan parce que les journées sont courtes et il se plaint d'avoir en permanence les pieds mouillés, de souffrir de rhume et d'asthme. L'hiver 1783-1784, particulièrement glacial, rendit impossible tout travail extérieur.

\section{Les clercs}

Les clercs travaillent à Angers et " en campagne ". Essentiellement chargés de mettre au propre les brouillons, ils doivent maîtriser suffisamment la calligraphie pour pouvoir en jouer dans la graphie des textes. Les terriers réalisés dans le cabinet de Labry sont effectivement d'une très grande qualité.

Lors de l'embauche, Labry passe avec ses clercs, tous originaires d'Angers, un contrat sous seing privé. Il nous en reste quinze, pour la période courant de décembre 1773 à décembre $1778^{71}$, soit 5 ans. Chacun s'engage à « aller où led. sieur Labry voudra [l']envoyer pour le représenter en tout ce qui concerne l'état de feudiste et [à se] comporter de façon que led. sieur Labry n'en puisse avoir aucun reproche ". La durée d'engagement varie de 1 à 3 ans; sur les six embauchés en 1778, deux le sont pour 2 ans et quatre pour 1 an seulement. À partir de décembre 1773, l'un d'eux doit assurer une fonction de chef de bureau chargé de veiller au bon fonctionnement du bureau en l'absence de son patron ${ }^{72}$. Les gages annuels, payables par quartier, vont de 100 à $500 \mathrm{lt}^{73}$. Les apprentis qui se forment dans le cabinet reçoivent 100 lt la première année et 300 lt pour la troisième et dernière année; les clercs touchent entre 300 et 500 lt. Le seuil de 300 lt marque

70. Arch. dép. de Maine-et-Loire, E 578. Dans le mémoire, toutes les superficies en boisselées ont été converties en perches et le résultat a été noté dans la marge.

71. Arch. dép. de Maine-et-Loire, E 2966. Labry recrute sans passer d'annonce dans les Affiches d'Angers.

72. L'épouse de Labry assure aussi différentes responsabilités dans le fonctionnement du cabinet quand son mari est " en campagne ", comme en témoigne leur correspondance.

73. En 1778, Labry embauche deux clercs pour des gages de 500 lt : l'un en janvier, pour deux ans, le second en août, pour un an. 
donc la différence entre un apprenti et un clerc. À Angers, ils sont "nourris, logés, blanchis ", en campagne, ils sont nourris et logés. Les clercs sont engagés " pour trois cents jours par an et huit heures chaque jour " quelle que soit la saison. La journée de travail, qui doit être le " temps de l'étude sans causer et sans s'amuser ", est " à commencer le matin entre sept et huit heures pour avoir fait quatre heures à midi et le soir entre une et deux pour avoir fini les quatre heures entre cinq et six heures". Dimanches et fêtes sont évidemment chômés; Labry lui-même respecte les fêtes mais moins strictement les dimanches. Le samedi, chaque clerc doit remettre à Labry, - en son absence à son épouse ou à un clerc ancien -, " un bordereau du temps employé pendant la semaine et ce à quoi il a travaillé ». Certains travaillent mal : en décembre 1785, Letourneau, qui œuvre alors pour le terrier d'Étiau à Vernantes, se plaint que la « recette a été des plus difficiles, d'une longueur à perdre patience, les tables tant des censifs que des livres de recette étant horriblement faites " et comportant d'innombrables confusions d'articles et de noms (d'autant que les homonymes sont nombreux) ${ }^{74}$.

L'effectif des clercs varie ${ }^{75}$, parfois d'une semaine sur l'autre; il atteint son maximum (11) en 1779 et en 1784-1785. Ses fluctuations ne semblent pas avoir de rapport étroit avec celles des clients ou même celles du travail accompli. Au printemps 1781, le nom de 14 clients apparaît dans le " semainier "; durant l'été il n'y en a plus que sept ou huit et au printemps suivant, seulement quatre, dont un seul vraiment important. À partir d'avril 1783, la quasi-totalité de l'activité est consacrée au terrier de Baugé et l'effectif des clercs remonte à 10 ou 11 entre août 1784 et mi-mars 1785. Parfois les clercs semblent presque inoccupés pendant plusieurs jours. Si quelques clercs font preuve d'une réelle stabilité, à l'instar de Macault qui resta presque dix ans (du 21 novembre 1779 au 18 août 1789), les départs et les nouvelles embauches se succèdent parfois rapidement.

Plusieurs clercs formés chez Labry se sont établis comme feudistes dans la décennie 1780, quelques-uns obtenant même de gros contrats. Labry s'était associé en 1775 avec son principal et plus ancien collaborateur, Aubin ${ }^{76}$; ils avaient signé ensemble le contrat pour la réfection du terrier du domaine de Baugé en février 1782. Après des années de collaboration efficace, de sérieuses dissensions se firent jour, Labry accusant Aubin de ne pas respecter le "traité de société " et Aubin contestant le calcul des sommes que Labry disait lui devoir. En 1786 Aubin décide d'" abandonne(r) la société " et son nom n'apparaît plus parmi ceux des clercs à partir du

74. Arch. dép. de Maine-et-Loire, E 2982. Il met en cause le travail de son collègue Moriceau, embauché un an auparavant.

75. Arch. dép. de Maine-et-Loire, E 2967. « Semainier où sont portées les journées de MM. mes clercs et pour qui ils les ont employées " 1775-1786.

76. Aubin était entré dans le cabinet de Labry le $1^{\text {er }}$ juillet 1771 comme clerc " pour travailler à la féodalité "; en janvier 1775, son contrat avait été prolongé et il " entra en société pour le sixième des profits de tous les fiefs " à compter du $1^{\text {er }}$ janvier 1775 , et cinq ans plus tard, le quart. En même temps, il était devenu greffier de la seigneurie de la Gouberie en mars 1775 et en juin 1779 de celle de Beauvais, la Coutancière, etc. 
5 novembre. Toutefois il poursuit sa carrière avec succès puisqu'il devient " feudiste et commissaire à terrier de Monsieur pour son château d'Angers et dépendances " et reçoit à ce titre en 1788 et 1789 les déclarations ${ }^{77}$; il travaille avec deux anciens collègues, qui s'intitulent aussi feudistes, en concurrence avec leur ancien patron, dont l'activité semble sur le déclin.

\section{Moyens financiers}

Dans les préoccupations de Labry, les soucis financiers occupent une place majeure. Sans cesse à la recherche d'argent, en particulier pour pouvoir payer ses clercs, il doit assumer de nombreux frais, qui lui sont, en principe, partiellement remboursés par ses clients : paiement des indicateurs et arpenteurs, salaire des clercs, coût des fournitures pour le cabinet, notes du relieur, location de chevaux, frais de voyage et d'hébergement, frais administratifs (rémunération des huissiers pour la convocation aux assises, pour les poursuites etc.), règlement du contrôle des actes, coût des actes notariés, expédition des titres. À Angers, les bureaux établis dans sa propre maison doivent être chauffés et bien éclairés puisque même en hiver la journée de travail est de 8 heures. Labry inscrit avec exactitude le prix des fournitures (papier, encre de Chine, poudre, plumes, crayons...). Le coût du papier est important : il en faut de grosses quantités, de qualités variées et donc de prix différents ${ }^{78}$. Les terriers sont constitués de cahiers reliés en volumes, tout comme les titres qui ne sont plus conservés en liasses. En 1784, selon une facture du relieur, la reliure d'un "grand volume " est de 9 lt, celle d'un petit 3 lt $^{79}$.

Dans les dépenses du cabinet et le prix du terrier, le coût de la maind'œuvre, même pour de petits fiefs, est le principal poste. Labry se fait payer 6 lt, quelquefois 8 , par jour et il demande pour ses clercs 4 lt; ce tarif reste stable tout au long de la période. Pour le fief de Saint-Martin-dela-Place, sur lequel les moines lèvent en particulier des terrages au $6^{\mathrm{e}}$, il a fallu environ 1250 jours de travail (51 mois de 25 jours ouvrés), soit à 4 lt/ jour, 5100 lt pour les clercs, sans compter le salaire de Labry (à 6 lt/jour); s'y ajoute le coût de l'entretien des hommes sur place. C'est tardivement qu'un tarif officiel est établi pour la rémunération des actes des feudistes; des lettres patentes du 10 août 1786 fixent " la taxe des droits des commissaires à terrier " selon la catégorie de l'acte (foi et hommage, aveux et dénombrements, déclarations censives, etc.) et selon la nature et la valeur du bien concerné, ou bien pour les censives, en application d'un forfait; la rémunération de l'écriture de chaque page est aussi prévue ${ }^{80}$. Sans doute ce tarif fut-il trop tardif pour être appliqué.

77. Arch. dép. de Maine-et-Loire, C 243 et $s q$.

78. Arch. dép. de Maine-et-Loire, E 2969.

79. Arch. dép. de Maine-et-Loire, E 2984. Les peaux utilisées sont le parchemin ou la basane; selon Bretonneau, cette dernière ne coûte pas beaucoup plus cher mais donne un meilleur résultat.

80. BnF, F-21217 (72) [consultable sur Gallica]. Pour une " déclaration censuelle " concernant un bien de campagne quelle que soit sa nature, il sera payé 15 sols pour le 
Les recettes sont difficiles à estimer car la comptabilité de Labry, telle qu'elle nous est parvenue, est souvent désordonnée et floue, sans l'esprit de précision qu'il manifeste dans la réalisation des terriers. Il reçoit des paiements de ses clients mais il conserve aussi, au titre du travail accompli, une partie des arrérages perçus au nom des seigneurs : en février 1774 c'est 804 lt pour "ventes " (on ignore pour quel seigneur et quelle terre); le 15 novembre il indique une recette d'assises sans plus de précision s'élevant à 165117 s 4 d; le 24 février, il inscrit 53119 s 8 d d'arrérages de rentes de la Poissonnière dues à madame de Million.

Rares sont les clients qui paient sans barguigner. Bien souvent Labry est " en avance " et il consacre beaucoup de temps à essayer de se faire payer : à l'été 1785, il fait la tournée de ses clients pour rentrer dans ses fonds, mais en vain. En décembre 1785, pour la réalisation du terrier de Baugé ${ }^{81}$, ses recettes (2 059 l 9 s 7 d) ne couvrent que la moitié des dépenses et mises ( 393019 s 9 d) mais " les grosses dépenses sont toutes faites et nous avons beaucoup de lods anciens à recouvrer, tant pour les mouvances entreprises par les seigneurs voisins que par le dépouillement des registres des bureaux du centième denier ${ }^{82}$. " Ces questions financières sont une source de tension entre Labry et ses clients; de multiples lettres en témoignent. Il est parfois accusé de malversation, car soupçonné de conserver indûment de l'argent, puisque dans ses comptes il ne précise pas toujours l'origine des recettes. Les récriminations financières des ecclésiastiques et des grands nobles, comme Harcourt ${ }^{83}$ ou Maillé La Tour-Landry, qui dans la décennie 1780 semblent être toujours à court d'argent, sont particulièrement acerbes et ils y ajoutent des critiques sur la qualité du travail et l'efficacité de Labry, ses promesses non tenues, la rétention des titres...

Autant les relations avec Sheridan, marquis d'Étiau, étaient cordiales, presque amicales comme le prouvent de nombreuses lettres ${ }^{84}$, autant elles sont tendues avec son second gendre, le comte de Maillé La Tour-Landry ${ }^{85}$.

premier article, 7 sols et demi pour les suivants. L'écriture des rôles sera payée " vingt sols par rôle en parchemin, à raison de vingt-deux lignes à la page et quinze syllabes à la ligne ». Il faut ajouter le coût du parchemin, du papier, ainsi que " les droits de scel et de contrôle, qui seront payés et remboursés par les vassaux et censitaires ".

81. Arch. dép. de Maine-et-Loire, E 2978. Lettre du 3 décembre 1785.

82. La consultation de ces registres permet de repérer les cessions de biens pour lesquelles les " ventes " n'ont pas été payées.

83. Id. . Charles Louis Hector (1743-1820), marquis d'Harcourt, est alors baron seigneur de Fontaine-Guérin, le Vieil-Baugé, Brion, Les Hayes, brigadier des armées du roi et commissaire général de la cavalerie de France. Bretonneau écrit à Labry le 23 décembre 1783 : «[...] assez communément on est fort à plaindre quand on a affaire à des grands seigneurs parce que le préjugé est presque toujours pour eux ".

84. Sheridan rémunère souvent Labry avec du vin : en juin 1773, trois barriques de vin de Bordeaux, plus apprécié que les vins angevins, pour 342118 s, et encore en juin 1775 pour 42216 s 3 d.; à Nantes, il peut s'en procurer aisément.

85. Hardy, intendant du marquis d'Harcourt, se plaint aussi de son maître qui exige, avec beaucoup d'insistance semble-t-il, de l'argent " [...] j'ai eu beaucoup de raisons avec lui jusqu'à lui demander mon compte " écrit-il à Aubin le 25 décembre 1782. En 1785, Letourneau relève les difficultés rencontrées dans son travail à Étiau et du caractère 
Le 24 janvier 1780, celui-ci lui annonce que, excédé par ses demandes d'argent, il va le remplacer par son " homme d'affaires qui entend très bien la féodalité, c'est lui [qu'il] compte charger de cette opération "; Labry doit donc lui remettre ses papiers ${ }^{86}$. En septembre 1783, il le soupçonne de détournements de fonds :

" Je trouve très surprenant que vous touchiez mes rentes et mes censifs et que vous ne m'en rendiez aucuns comptes. On ne sait trop quel nom donner à cette conduite qui vous est familière. Aiez la bonté, Monsieur, de m'apporter tout l'argent que vous avez touché pour moi, et de m'en rendre un compte exact; lorsque je serai établi à Jalesnes, je vous préviendrai d'y venir également justifier votre travail en me remettant les rentrées qui vous ont été faites, et les papiers de Jalesnes et Poligné, ne voulant avoir à faire à vous d'aucune manière ${ }^{87}$. "

En 1782, Le Jumeau, baron de Blou, s'étonne : «Vous ne m’avez donné aucune connaissance [du travail fait] sinon que je vois que tout n'a servi qu'à vous faire payer toutes mes rentes, ventes, rachats, etc. et je n'en suis pas plus avancé puisque vous avez tout touché, que je n'ai plus touché un liard "; le 20 février 1784, il insiste : "Tout le monde m'assure que vous avez dû recevoir à moi plus de dix mille livres. Donnez-moi un état de ce que vous avez fait ${ }^{88}$."

Autres clients difficiles, les ecclésiastiques. Devant le coût des opérations $^{89}$, les religieux de l'abbaye de Saint-Florent de Saumur ou de SaintNicolas d'Angers ont vivement protesté ${ }^{90}$. Le procureur de cette dernière reconnaît le 26 août 1775 qu'en s'engageant dans la rénovation du terrier " il a fait une entreprise ruineuse et qui n'a produit à notre maison qu'un avantage bien léger ${ }^{91}$ ". Le prieur de Saint-Florent répète que la maison est " sans le sou et dans la plus grande gêne " (mars 1779), ce dont Labry n'a cure : "Vous demandez cinquante louis comme vous demandez un liard ${ }^{92}$ "; il l'accuse d'exagérer le coût du travail : «Quand j'en parle à des séculiers, gens qui connaissent les fiefs et que je leur dis que vous demandez encore, ils ne peuvent pas le concevoir. " Labry réclame au moins le versement d'acomptes " ne sachant comment [se] retourner" car il doit payer ses clercs " ne [lui] faisant point crédit ", d'autant que s'il a accompli les trois

acariâtre du marquis : " Je n'ai pas lieu d'en être fort content : toujours des propos fort déplacés, je suis très satisfait de ne pas lui remettre l'ouvrage. "

86. Arch. dép. de Maine-et-Loire, E 2982.

87. Arch. dép. de Maine-et-Loire, E 2983. Un peu plus tard, une violente altercation éclate entre les deux hommes. Labry est accusé d'avoir " pris au collet " le comte, ce qu'il nie farouchement. Une transaction financière fut conclue (Id., E 2971/2).

88. Arch. dép. de Maine-et-Loire, E 2977. Lettre du 26 septembre 1782.

89. Voir supra.

90. La facture la plus lourde fut pour l'abbaye de Saint-Maur mais on ignore quelles réactions elle suscita.

91. Arch. dép. de Maine-et-Loire, E 2980.

92. Arch. dép. de Maine-et-Loire, E 2980. 
quarts du travail il n'a été payé que pour un quart ${ }^{93}$; il affirme avoir dû emprunter pour "ne pas gêner " les moines. Les sommes en cause sont élevées : selon ses comptes du 25 juin 1777, les dépenses se montent à 5175 lt et les impayés à 3095 lt, soit $60 \%$ du total. Il ne peut aller plus vite dans son travail : pour les titres : " il faut du temps pour mettre en ordre une telle quantité de papiers " et " c'est la longueur du travail qui fait qu'il m'est dû beaucoup " : Labry résume bien en quelques mots l'ampleur de la tâche à accomplir.

Dans la décennie 1780 il a sans doute trop de gros chantiers en cours; de nombreux clients lui reprochent de ne pas travailler assez vite sur leurs affaires et de les lanterner. M. de la Lorie se plaint en août 1784 : "Vous m'aviez dit que vous viendriez ici à Carême; on ne vous a point vu ${ }^{94}$ "; au même moment Bretonneau l'apostrophe : "Avez-vous donc juré de ne plus venir ici, êtes-vous monté aux cieux, ou plus vraisemblablement descendu aux enfers ? ${ }^{95}$ ". Boisjourdan lui écrit en mars 1789 que ses affaires n'avancent toujours pas " depuis cinq ans que vous me tenez en esclavage ${ }^{96}$ ".

\section{Les opérations de rénovation d'un terrier}

Dans le ressort des coutumes qui proclament l'imprescriptibilité du cens, un terrier est susceptible d'être renouvelé tous les 30 ans. Selon la définition très concise de La Poix de Fréminville, un terrier est " un livre, registre ou cartulaire qui renferme les lois de la seigneurie ${ }^{97}$ ". Ferrières est plus précis : " [c'est une] description de tous les héritages, tant féodaux que roturiers, qui sont dans la mouvance féodale d'un seigneur ou dans sa censive, et de tous les droits, dixmes, terrages, coutumes, corvées, rentes foncières, seigneuriales ou non seigneuriales, et autres semblables, de tous les vassaux et arrières-vassaux et sujets censiers, et tenanciers à autres droits [...]. Les déclarations des vassaux et censitaires portées par les terriers font titres contre eux en faveur du seigneur ${ }^{98}$. "Tous les vassaux et sujets de la seigneurie sont donc concernés quel que soit leur statut juridique et social; ainsi, en 1782, les commissaires à terrier du duc de Luynes demandent au marquis d'Étiau l'aveu de la châtellenie de Pocé à Vernoil ${ }^{99}$.

93. Arch. dép. de Maine-et-Loire, E 2983. Lettre en date du 25 juin 1777. La série des lettres conservées s'arrête à cette date; le dénouement du litige n'est donc pas connu. Les difficultés financières des moines ont une autre origine : dans ces mêmes années ils ont engagé une très coûteuse reconstruction des bâtiments monastiques. Ils emploient, sans doute depuis 1778, au moins un autre feudiste, Richou, notaire royal à Saulgé-L'Hôpital, " commissaire nommé pour la confection des terriers de lad. abbaye " (Id., E 3175).

94. Arch. dép. de Maine-et-Loire, E 2989.

95. Arch. dép. de Maine-et-Loire, E 2978.

96. Arch. dép. de Maine-et-Loire, E 2977.

97. FreminVILLE, Edme LA PoIX DE, La pratique universelle..., op. cit., t. 1, 1746, p. 61.

98. FERrIÈRE, Claude Joseph DE, Dictionnaire de droit et de pratique, Paris, chez la Ve Savoye, nouvelle éd., 1771, t. II, p. 311.

99. Arch. dép. de Maine-et-Loire, E 2980. 


\section{Les particularismes de l'Anjou}

En droit, un terrier est un document « authentique " qu'il est impossible de corriger; il se périme très vite puisqu'on ne peut y porter les changements de propriétaire et les modifications des limites de propriété; pour qu'il reste utilisable un seigneur attentif à ses droits doit donc le faire refaire régulièrement. La situation est compliquée en Anjou par le statut original de nombreuses terres, qui sont des "frèches " : il s'agit de " rentes solidairement dues à un seigneur par les différents propriétaires d'un fonds ${ }^{100}$ "; les terres sont détenues par des individus, les co-frécheurs, qui sont solidairement responsables du paiement du cens. Mutations de propriété et partages successoraux entraînent sans cesse des modifications; l'Anjou étant une terre de partage roturier strictement égalitaire, à chaque génération la fragmentation des propriétés s'aggrave ${ }^{101}$. Les frécheurs peuvent en profiter pour ne pas payer : en 1780 le fief de la Forêt de Vernantes, relevant de Jalesnes, composé de 368 frèches, " qui n'a jamais été bien tenu et dont les sujets ne payaient presque pas, pouvait rapporter $4734112 \mathrm{~s} 2 \mathrm{~d}$ suivant le relevé qui en a été fait sur le papier censif ${ }^{102}$ ». Il faut régulièrement remettre à jour et en ordre la liste des propriétaires, et éventuellement rappeler des droits oubliés, opérations qui sont réalisées lorsque les censitaires font leurs déclarations au cours des assises, procédure qui est aussi une particularité de l'Anjou, du Maine et de la Touraine (voir ci-après).

En outre, en avril 1771, les duché d'Anjou et comté du Maine furent concédés en apanage au comte de Provence, par l'" édit du roi portant lettres d'apanage de Louis Stanislas Xavier, petit-fils de France ${ }^{103}$ ", décision qui ouvrit de vastes perspectives aux feudistes angevins ${ }^{104}$ ! Les lettres patentes du prince des 13 février et 4 juillet 1773 ordonnent en effet que

100. Arch. dép. de Maine-et-Loire, C 101. Lettre de l'intendant Du Cluzel (12 septembre 1769). Selon Claude Pocquet de Livonnière, " ces sortes de rentes (sont) très-communes en Anjou [...] Le terme de Frarescheurs, ou Frescheurs se prend souvent dans notre coutume pour cohéritier [...] Or, [...] il arrive ordinairement que l'héritage du père chargé d'une Rente se divise entre les enfants, frères et sœurs, que chacun possédant une partie de l'héritage est tenu solidairement de la totalité de la Rente, quoiqu'il n'en doive personnellement que sa part \& portion. " (Traité des fiefs, Paris, P.G. Le Mercier, 1756 (4e éd.) [1729], p. 540 .

101. Arch. dép. de Maine-et-Loire, E 501, fol. 1. Ainsi la "grande frèche du clos de SousCoins ", à Murs, comprend 35 quartiers de vigne que se partagent 39 cofrécheurs, le plus petit lopin étant de $1 / 8^{\mathrm{e}}$ de quartier (0,02 ha). Elle doit 2 setiers 7 boisseaux de seigle mesure de Brissac et $3 \mathrm{~s} 6 \mathrm{~d}$ de cens.

102. Arch. dép. de Maine-et-Loire, E 2968.

103. Arch. dép. de Maine-et-Loire, C 217. Lettres patentes du roi d'avril 1771. " Ces lettres patentes créant l'apanage [...] permettent [au comte d'Artois] de mettre en place une véritable administration qui veille avec une grande rigueur au versement régulier de tous les revenus des terres et des droits possédés " (Malllard, Jacques, Histoire de l'Anjou. L'Ancien Régime et la Révolution en Anjou, Paris, Picard, 2011, p. 25).

104. Affiches d'Angers, 12 novembre 1773, p. 7-8. Les feudistes d'Angers ne manquent pas alors de proposer leurs services : le 3 mars 1775, les Affiches d'Angers publient une annonce du sieur Fombeux à l'intention des seigneurs « qui relèvent de l'apanage ne pouvant se dispenser de faire tenir leurs assises afin de rendre des aveux hors de critique ". 
" tous les vassaux de l'apanage, tant laïques qu'ecclésiastiques, sans exception, seraient tenus de rendre dans les délais y fixés ès mains du chancelier de Monsieur les foy et hommage-lige qu'ils doivent pour les terres, fiefs et seigneuries qu'ils possèdent dans la mouvance du duché d'Anjou et comté du Maine, de fournir les aveux et dénombrements et déclarations de temporel, dans les délais fixés par les coutumes, après les foy et hommages faits et duement enregistrés, et à défaut par lesd. vassaux de satisfaire au contenu desd. lettres, qu'ils seraient poursuivis [...] par la voie de saisie féodale, avec établissement de commissaires, ainsi qu'il est prescrit par les coutumes ${ }^{105}$ ".

Des assignations à comparaître sont envoyées dans la fin de l'année $1774^{106}$. De plus, le 15 juin 1782, le prince en son conseil délivre " des lettres patentes en forme de lettres à terrier ", pour la baronnie de Baugé; Bretonneau, receveur de ces terres et commissaire à terrier, s'associe avec Labry et Aubin pour réaliser le terrier; des assises sont ensuite tenues ${ }^{107}$. Pour le " château d'Angers et dépendances ", les lettres à terrier datent seulement du 8 janvier $1788^{108}$.

\section{Méthodes et pratiques}

La procédure à suivre est précise. Il faut obtenir du roi, qui est seul à pouvoir en délivrer, des "lettres de papier terrier en chancellerie ", théoriquement obligatoires ${ }^{109}$, " pour faire appeler par-devant notaire à ce commis tous les débiteurs des redevances prétendues par les seigneurs afin de les connaître et d'en passer forme authentique ". Elles doivent être enregistrées par le tribunal du lieu; Bretonneau reçoit les lettres à terrier pour la baronnie de Baugé en août 1782, mais il se heurte pour leur enregistrement à l'obstruction des officiers de la sénéchaussée de Baugé ${ }^{110}$. Le seigneur requérant fait alors faire les proclamations utiles par cri public et affichage; ensuite les assises peuvent s'ouvrir : en effet, en Anjou, ce n'est pas un notaire ès qualité qui instrumente. Quand le terrier est achevé, le juge qui a entériné les lettres à terrier le clôt, puis les actes passent au contrôle des actes. Si dans les archives de Labry il est rarement question de telles lettres, c'est que selon Fréminville, elles ne sont pas absolument obligatoires " en ce qu'il est de droit commun qu'un seigneur peut se faire reconnaître les droits qui lui sont dus par ces sortes d'actes " mais il " est absolument nécessaire d'en obtenir pour une seigneurie qui a haute justice. C'est ce qui a passé en usage dans tout le royaume ${ }^{111}$ ".

105. Arch. dép. de Maine-et-Loire, C 254 (circulaire du bureau des finances de Tours).

106. Id. Assignation délivrée au sieur Leloir, habitant Corzé, 15 novembre 1774.

107. Id.

108. Arch. dép. de Maine-et-Loire, C 243.

109. Les seigneurs ecclésiastiques ont été dispensés de cette obligation par l'ordonnance de Blois (art. 4) en 1579 et l'édit de Melun (art. 26) en 1580 (cf. SoBoul, Albert, « De la pratique des terriers à la veille de la Révolution ", Annales ESC, nov.-déc. 1964, nº 6, p. 1052). 110. Arch. dép. de Maine-et-Loire, 5 E2978.

111. FréminVILLE, Edme LA PoIX DE, La pratique universelle..., op. cit., t. 1, p. 62-64. 
Dans un mémoire ${ }^{112}$, Labry a énoncé clairement chacune des opérations nécessaires pour la rénovation du terrier, composé de plus de 30 volumes, de la terre de Saint-Martin-de-la-Place dont le fief comporte plus de 5000 articles, Labry estime qu'il faudra 51 mois de travail, soit 17 mois de travail pour trois hommes, sans compter "les circonstances imprévues ", comme des contestations, "ce qui est inévitable dans une tenue d'assises " :

1- « Examen, étictement des titres pour ceux qui ne le sont pas, les articuler puisqu'ils ne le sont pas pour la majeure partie, mettre d'ordre, porter aux tables personnelles les noms des contractants depuis 1760 jusqu'à 1779 , porter à la table les folios et les articles des titres, lesquels ont été obmis, pour toutes ces opérations il convient d'employer au moins trois mois [d'une personne]

3 mois

2- le relevé des cintures des fresches ${ }^{113}$ et le nom des débiteurs d'icelles, tant au livre du f[ermier] de MM. les religieux que celui de M. l'abbé, faire la copie du terrier [existant], le tout pour former une base du dépouillement des titres et faire icelui dépouillement, pour à ce parvenir il sera employé quinze mois

15 mois 3 - la prise des confrontations et connaissances locales, trois mois 3 mois 4 - le placement ou adoption des titres au plan, pour trois mois au moins

3 mois

5 - pour procéder aux feuilles ou projets de déclaration de chaque sujet à 60 articles par jour à un seul homme forme cinq mois puisqu'il y a environ 6000 articles dans le fief 5 mois 6 - l'assise qui ne peut se tenir qu'il n'y ait au moins 3 hommes*, on ne peut guère être à moins de trois mois, ce qui forme neuf mois 9 mois 7 - la remise d'assises pendant un mois à deux hommes 2 mois 8 - différents mémoires, s'il se trouve des contestations (ce qui est inévitable dans une tenue d'assises) et demandes à ce sujet, temps imprévu imprévu 9 - étictement de la tenue d'assises qui sera faite, les tables personnelles et réelles, au moins deux mois 2 mois 10 - pour faire le projet de censif, trois mois 3 mois 11 - mettre ce projet au net, rapporter les titres au soutien de chaque objet, faire les tables personnelles et réelles d'icelui, six mois 6 mois

* laquelle peut être tenue dans le courant de 1780 ou 1781.

Cinq phases principales ${ }^{114}$, toutes très longues, apparaissent : la mise en ordre des titres, la levée des plans, la tenue des assises, la réalisation d'un brouillon, enfin la mise au net et la confection du terrier définitif, qui se fait

112. Arch. dép. de Maine-et-Loire, H 2417. Sur ce territoire qui dépend de l'abbaye SaintFlorent de Saumur les moines ont le droit de lever un terrage de $1 / 6^{\mathrm{e}}$, comme l'atteste un terrier réalisé en 1681. En 1777, s'élève une contestation; c'est peut-être la raison qui amène les moines à commander un nouveau terrier.

113. Sur les plans les frèches apparaissent très souvent comme de vastes parcelles de terre subdivisées en lanières parallèles, très étroites, dont les limites ne pouvant être matérialisées sont figurées par un simple trait; elles s'allongent le plus souvent entre deux chemins.

114. Les opérations pour des clients laïques sont évidemment du même ordre (cf. Arch. dép. de Maine-et-Loire, E 2971/2, "État de ce qu'il y a à faire aux fiefs du marquisat d'Étiau ", du 26 juin 1780, détaillé par BÉchu, Philippe, "Un feudiste... ", loc. cit., p. 224). 
à partir d'un " projet " ou brouillon, lequel est le plus souvent la copie d'un censif plus ancien qui est mis à jour grâce aux déclarations des tenanciers et aux plans levés ${ }^{115}$.

Tous les contrats passés par Labry insistent sur la première obligation : mettre de l'ordre! Alors que la plus grande confusion semble avoir régné depuis longtemps dans les chartriers, dans la seconde moitié du XVIII ${ }^{\mathrm{e}}$ siècle de nombreux seigneurs ne supportent plus cette situation. En décembre 1783, Boisjourdan, à Grez-Neuville ${ }^{116}$, informe " monsieur de La Brïe (sic) " qu'avant de lui envoyer ses titres il les a fait ranger : "C'était un cahos (sic) où il a fallu du temps pour débrouiller ". Labry met en garde les moines de Saint-Nicolas à Angers le 29 août 1775 : " Je vous répète encore que tant que votre chartrier commun ne sera pas plus en règle qu'il l'est, que l'on fera toujours de la bouillie pour les chats (sic); il entrait bien dans votre projet de le faire mettre en ordre et ce qui vous retenait était que l'abbé en devait faire les frais ", mais comme ce dernier a refusé de payer, " nous avons toujours eu l'un et l'autre le bec dans l'eau (sic) [...] Si vous ne faites arranger le chartrier commun que quand vos abbés voudront y contribuer, il restera encore quelques siècles dans l'état où il est ${ }^{117}$. "

Il faut classer, sans en jeter aucun, les titres, anciens et nouveaux, les inventorier et les faire relier en registres factices, en y insérant des tables personnelles et réelles afin d'en faciliter la consultation. Cette mise en ordre peut relever de la gageure étant donné la complexité des structures féodaloseigneuriales, que les traités publiés au XVIII ${ }^{\mathrm{e}}$ siècle mettent bien en avant et qui peut être la source d'une forte litigiosité.

Autre étape obligatoire puisque les coordonnées géographiques et le numéro de chaque parcelle doivent figurer dans la marge des reconnaissances pour permettre de l'identifier : la levée de plans. Il est désormais évident qu'un terrier doit comporter des plans; rares jusqu'alors, ceux-ci se multiplient après $1740^{118}$. L'esprit du siècle exige précision et clarté : « Les plans-terriers de la seconde moitié du XVIII ${ }^{\mathrm{e}}$ siècle se caractérisent par leur précision et leur lisibilité, la correspondance fiable avec les déclarations des censitaires, la méthode " géométrique " qui l'emporte sur la méthode " visuelle " et l'emploi de nouveaux outils de mesure ${ }^{119}$. " Parfois regroupés en atlas, ils sont d'une grande qualité graphique. Ils assurent une plus grande valeur juridique au terrier et peuvent donner aux seigneurs le plaisir

115. Les lettres patentes du 20 août 1786 (BnF, F-21217 [72]) prévoient (art. XIII) que les minutes des terriers achevés devront être remis au greffe des sièges royaux et qu'une " grosse " devra en être présentée au greffier qui en réalisera une " collation ", avant d'être déposée dans les archives du seigneur. Le feudiste devrait donc faire réaliser une copie. 116. Arch. dép. de Maine-et-Loire, E 2977.

117. Arch. dép. de Maine-et-Loire, E 2980. Lettre au procureur de l'abbaye.

118. Soboul, Albert, " De la pratique des terriers... ", loc. cit., p. 1054.

119. BIANCHI, Serge, "Terriers, plans-terriers et Révolution ", in BRUNEL, Ghislain, GuYotJEAnNin, Olivier et alii, éd., Terriers et plans-terriers..., op. cit., p. 313. 
et la satisfaction de contempler " l'étendue de leurs domaines ${ }^{120}$ ». Les plans doivent être réalisés avant la tenue des assises et l'opération prend du temps ${ }^{121}$ : après avoir relevé les confronts des terres selon les déclarations des indicateurs, le géomètre établit successivement deux types de plans : " le visuel qui se lève à la vue, qui est toujours irrégulier, et le géométrique qui se lève avec des instruments, qui est régulier " selon Fréminville; ainsi, le feudiste peut avoir " le plan des lieux de chaque canton, pour faire dans sa chambre le placement et la distribution de chaque article sur les héritages qu'ils affectent ${ }^{122}$ ». Ensuite se déroulent les assises, puis le brouillon du terrier est réalisé et, mis au net après correction, permet l'établissement du papier terrier définitif.

Malgré les progrès réalisés il n'existe pas de méthode de travail qui s'impose à tous et chaque feudiste a la sienne; ainsi s'expliquent les différences observables dans l'organisation et la présentation des terriers ${ }^{123}$. Toutefois, les feudistes du XVIII ${ }^{\mathrm{e}}$ siècle sont soucieux de la valeur juridique et de l'utilisation pratique des documents qu'ils produisent. Les plus méthodiques comme Labry consacrent dans les registres nouveaux une page par article; ils y notent, après la description du lieu et l'intitulé du " propriétaire ", " les titres au soutien ", parfois très anciens, par ordre chronologique et laissent de grands espaces blancs pour y porter les titres ultérieurs et de vastes marges pour " moderniser les vassaux et les sujets [...] au moyen de quoi ce censif pourra servir pendant un siècle, après lequel il ne faudra que le moderniser ${ }^{124}$ ». Pour que le terrier reste facilement manipulable et ne s'abîme pas trop vite, ses registres doivent être solidement reliés et n'être pas trop gros ${ }^{125}$.

120. Antolne, Annie, Le paysage de l'historien. Archéologie des bocages de l'Ouest de la France à l'époque moderne, Rennes, PUR, 2002, p. 94-95.

121. En 1769, G. DOYEN écrit que « Les premiers plans ont commencé dans cette province de Beauce vers la fin du seizième siècle [...] ; ils ne sont que visuels et très mal faits; on continua de cette façon jusques vers 1650 , que l'on commença de les faire en grandes feuilles de parchemin, cependant visuels comme les autres, mais moins mal faits. C'est au commencement de ce siècle que les plans géométriques ont pris naissance; \& il n'y a pas plus de trente ans que l'on connaît le propre usage d'un plan, c'est-à-dire que l'on sait que c'est par son seul moyen qu'on peut bien faire la rénovation d'un terrier " (Géométrie de l'arpenteur..., op. cit., p. 120). Doyen assigne à son manuel un but précis et limité; il insiste sur la technique de levée des limites de parcelles et la réalisation de plans de parcellaires ruraux (p. 121-182). Selon un feudiste du Bas-Maine, Duchemin de Villiers, l'usage de faire " dresser des cartes topographiques " de la seigneurie y est venu d'Anjou à partir de la moitié du XVIII ${ }^{\mathrm{e}}$ siècle (AnTOINE, Annie, Fiefs et villages du Bas-Maine au XVII ${ }^{e}$ siècle, Mayenne, ERO, 1994. p. 239).

122. FRÉMINVILle, Edme LA POIX DE, La pratique universelle..., op. cit., p. 113.

123. Pour le détail des méthodes de classement et de constitution des registres factices, voir Massala, Corinne, "Les feudistes angevins, ancêtres des archivistes ", dans MARAIS, Jean-Luc (dir.), Historiens de l'Anjou, Rennes, PUR, 2012, p. 81-87.

124. Arch. dép. de Maine-et-Loire, 1E 278. Projet de censif du fief d’Étiau (1781) par Desportes et Maillard.

125. Le livre des seigneurs..., op. cit., p. 156-157. Chaque volume ne doit pas avoir plus de 800 pages : "Un trop gros registre se fatigue beaucoup plus qu'un registre de taille proportionnée. On porte l'un volontiers, on traîne celui qui est trop lourd ». 
Ce travail est donc chronophage, même pour de petites seigneuries; il produit aussi des masses de papiers, mais il est indispensable pour assurer la qualité du résultat. Le feudiste doit donc être soigneusement choisi et les auteurs de traités spécialisés conseillent de ne pas regarder à la dépense : de petites économies peuvent naître de gros problèmes dus à des titres mal enregistrés, des plans imprécis... Loin de remettre en cause les droits du seigneur la rénovation permet de les conforter en améliorant la qualité des archives et en facilitant leur consultation. Toutefois un seigneur peut hésiter devant le coût de l'opération car, même si les déclarations des vassaux et sujets se font aux frais de ceux-ci, il dépasse le bénéfice financier qui peut en résulter. Dans les Affiches d'Angers du 3 mars 1775, un " avis intéressant les seigneurs de fiefs " déclare : "Les longues opérations jointes aux frais considérables qui jusqu'ici ont été inséparables de l'arrangement des archives et de la rénovation des terriers [sont] des obstacles capables d'éloigner les seigneurs du dessein de faire arranger leurs fiefs ".

\section{L'importance des titres}

Tous les " titres au soutien " doivent être cités de façon précise et exacte, que ce soit des déclarations, des actes d'acquêts, de partages, d'échanges, des ventes par décret... Il faut en noter la date et la nature dans le nouveau terrier. Dans certains chartriers, en particulier ceux des institutions ecclésiastiques, les collections sont massives : en 1778, pour les " papiers concernant Nantilly [appartenant à Saint-Florent de Saumur] arrangés et mis en volume comme ceux de Varrains [...] il y en avait pesant deux mille soixante quatre livres ${ }^{126}$ ". Les pièces datant du $\mathrm{XV}^{\mathrm{e}}$ siècle ne sont pas rares : pour un projet d'aveu pour la seigneurie des Grand et Petit Launay, Labry cite cinq documents du $\mathrm{XV}^{\mathrm{e}}$ siècle, dont un aveu daté de 1436, et six du $\mathrm{XVI}^{\mathrm{e}}$ siècle $^{127}$.

Pour travailler, le feudiste et ses clercs ont besoin de disposer des papiers et titres au cabinet, dont les locaux doivent être suffisamment vastes pour accueillir les titres des terres et fiefs mais aussi pour abriter les tables de travail des clercs. Les seigneurs attentifs à la gestion de leurs affaires et surtout de leurs finances sont extrêmement soucieux de la bonne conservation de ces documents et s'en séparent difficilement. Un seigneur résidant au Lion-d'Angers affirme en mars 1783 que "Les titres sont sous clé et nul ne peut les donner que moi ", que quelques-uns " ne doivent pas sortir du trésor " et que certains sont dans l'armoire à linge ${ }^{128}$. Pour le transport, il est fait le plus souvent appel à la messagerie comme celles de Beaufort, de Saumur... De Baugé, le 19 novembre 1782, Bretonneau informe Labry qu'il a " chargé au messager la malle et les deux paniers tout remplis des papiers qui étaient ici et que vous préférez de faire travailler chez

126. Arch. dép. de Maine-et-Loire, E 2980.

127. Arch. dép. de Maine-et-Loire, E 578.

128. Arch. dép. de Maine-et-Loire, E 2979. 
vous $^{129}$ "; plus tard, il expédie des papiers extraits du palais de justice de Baugé, tout en précisant qu' «ils sont bien gâtés mais il faut en tirer ce que l'on pourra " et qu'il a été difficile de les obtenir : "On [i.e. le lieutenant général de la sénéchaussée] est décidé à ne pas permettre le déplacement des titres qui sont au Palais ${ }^{130}$. " Le transport peut être confié à une personne privée, pour des raisons de sûreté, d'économie ${ }^{131}$, ou même d'opportunité : ainsi le temps de la foire Saint-Martin d'Angers, très fréquentée, permet d'avoir recours à des voisins complaisants ${ }^{132}$. Il faut prendre des précautions : envelopper les dossiers dans de la toile cirée pour les " parer [...] de tout événement ${ }^{133}$ ", faire fabriquer spécialement des boîtes et préciser au messager la nature de l'envoi " afin qu'on ait plus d'attention ".

Le feudiste ne doit pas conserver ces titres; en particulier dans les années 1780 , décennie qui voit les relations se tendre, les seigneurs les lui réclament souvent, affirmant en avoir besoin pour faire respecter leurs droits et surtout se faire payer leurs arrérages. En décembre 1776, le procureur de l'abbaye Saint-Nicolas rappelle : " [...] les livres que vous avez me sont nécessaires pour m'instruire de ce que j'ai à demander d'arrérages de cens et rentes que je voudrais bien faire rentrer pendant ce carême ${ }^{134}$ ". Le 26 septembre 1782, Le Jumeau, baron de Blou, s'emporte : "Voilà la Saint-Michel, temps où on fait payer les rentes. Avant que vous eussiez travaillé à mes assises, je me faisais payer, du moins la plus grande partie de celles qui me sont dues. Vous vous êtes emparé de tous mes titres sous le prétexte de me mettre en état de percevoir jusques au moindre censif. Je suis par ce moyen moins en état que jamais et ne sais plus par où m'y prendre [...]. Je veux mes titres dans le courant de la prochaine semaine afin de me faire payer [tant] mes rentes féodales que foncières dont vous avez tous les titres ${ }^{135}$."

L'accumulation et le classement de pièces écrites de nature variée mais très souvent notariées caractérisent les chartriers bien tenus. L'écrit prime incontestablement : le témoignage oral ne suffit plus, depuis longtemps, à prouver l'existence de droits. Pour étayer leurs déclarations aux assises, les censitaires doivent obligatoirement présenter ces titres très variés et parfois très anciens. Lors d'une procédure judiciaire les seigneurs se trouvent, grâce à leurs archives, en position de force face aux paysans, dont les titres sont généralement incomplets, détériorés ou perdus. En février 1790, les habitants de Cheffes protestent contre les usurpations de communaux et

129. Arch. dép. de Maine-et-Loire, E 2978.

130. Arch. dép. de Maine-et-Loire, E 2982.

131. Arch. dép. de Maine-et-Loire, E 2482. En septembre 1786, le baron de Blou demande à Labry de suivre une voie plus rapide et moins coûteuse que la messagerie pour lui renvoyer son terrier.

132. Arch. dép. de Maine-et-Loire, E 2973, lettre de La Motte de Croutelles en Poitou du 14 novembre 1774 .

133. Arch. dép. de Maine-et-Loire, E 2973, lettre de Dulis du 20 août 1775.

134. Arch. dép. de Maine-et-Loire, E 2980.

135. Arch. dép. de Maine-et-Loire, E 2977. 
écrivent : "Comment les paroissiens de Cheffes ont-ils pu se défendre? ils n'avaient pas les titres qui constatent leurs droits de propriété sur les communaux; ces titres, égarés ou recélés, tout favorisait les prétentions du seigneur du fief ${ }^{136}$."

\section{Les assises seigneuriales en Anjou}

\section{Une étape obligatoire}

"Suivant les coutumes du Maine de l'Anjou et de la Touraine, les seigneurs ont le droit de faire recevoir judiciairement par les officiers de leurs seigneuries les obéissances de leurs vassaux [...] les aveux, actes de foi et hommage, déclarations et autres reçus et attestés judiciairement, vulgairement appelés remembrances [...] produisant par un long usage autorisé par les coutumes le même effet que lesd. actes passés devant notaire ${ }^{137}$. "C'est une originalité en France ${ }^{138}$, comme le note Fréminville : " Il y a quelques provinces dans le royaume où les seigneurs font reconnaître leurs droits seigneuriaux dans [leurs] assises, où chaque censitaire fait sa déclaration et reconnaît les cens et devoirs qu'il doit à son seigneur sur les héritages qu'il possède ${ }^{139}$. " C'est d'ailleurs la principale compétence de cette justice domaniale; il en reste de très abondantes traces dans les archives seigneuriales. En Anjou, comme dans le Maine ${ }^{140}$ et la Touraine, déclarations et reconnaissances se font donc dans un cadre ordonné et collectif contraignant, celui des assises seigneuriales. En conséquence, les déclarations prennent la forme d'actes judiciaires tandis qu'ailleurs en France, elles constituent des actes notariés.

Selon l'article 1 de la coutume d'Anjou « basse justice, justice foncière, et simple voirie [sont] tout un ". Pour faire reconnaître ses droits, tout seigneur ${ }^{141}$ peut faire tenir des assises, "séances, ordinairement pour l'administration de la Justice; dans l'usage présent, on appelle de ce nom les

136. SAGnac, Philippe et Coron, P., Les comités..., op. cit., p. 143.

137. BnF, F-23650 (569) [consultable sur Gallica]. Arrest du conseil du Roi du 30 avril1718. 138. DONTENWILL, Serge, "Le rôle des assises et règlements de seigneurie dans la régulation sociale aux XVII ${ }^{\mathrm{e}}$ et XVIII ${ }^{\mathrm{e}}$ siècles. Le cas du Centre-Est de la France ", in BRIZAY, François, Follain, Antoine et SARRAZIN, Véronique (dir.), Les justices de village..., op. cit., p. 221-225. Cependant toutes les assises seigneuriales ont un rôle identique, exercer un contrôle sur les hommes et le territoire qu'est la zone de juridiction du seigneur.

139. FrÉminville, Edme LA POIX DE, Les vrais principes..., op. cit., t. 1, p. 83. Fréminville, critique envers les officiers seigneuriaux, juge préférable que les " reconnaissances, les remembrances et les obéissances dues aux seigneurs se [fassent] devant des notaires royaux [...] les officiers du seigneur étant et devant être regardés comme suspects". Dans le Traité des fiefs, Pocquet de Livonnière n'évoque jamais les assises, alors que leur organisation est une activité essentielle pour les feudistes angevins.

140. AnToIne, Annie, Fiefs et villages... op. cit., p. 240-249. Les coutumes de ces deux provinces sont très proches.

141. Antoine, Annie, "Justice foncière et contrôle social dans le Maine, l'Anjou et la Bretagne au XVIII ${ }^{\mathrm{e}}$ siècle ", in BRIZAY, François, Follain, Antoine et SARRAZIN, Véronique (dir.), Les justices de village..., op. cit., p. 269-284. 
séances relatives à la conservation des droits de fief des Seigneurs ${ }^{142}$ ". Il faut s'y " s'avouer sujet censivement et roturièrement ", " exhiber ses titres et rendre ses aveux et déclarations censives [pour les terres] qui relèvent des fiefs" et payer en outre les droits féodaux et censuels qui sont dus ${ }^{143}$. Les assises permettent aussi au seigneur de remplir ses obligations envers son seigneur supérieur : "Je vous aurai une vraie obligation de finir le plus tôt possible la tenue d'assises afin de me mettre en état de rendre mon aveu au terme de l'ordonnance et de faire l'éventillation de ce qui relève de Mgr le comte de Provence pour rendre ma foy et hommage dans le mois de may ou juin " écrit le seigneur de la Gouberie à Labry le 9 avril $1774^{144}$.

Cette tenue d'assises facilite le travail du feudiste : les sujets ne se présentent pas en ordre dispersé, les déclarations suivent toutes le même schéma et sont conservées ensemble dans le chartrier. Les assises permettent aussi éventuellement d'éviter une prescription trentenaire, les documents étant datés : en août 1784, un client de Labry lui demande de vérifier "si les rentiers ne pourraient point prescrire faute d'être réassignés, [... en effet] il paraît suivant la tenue d'assises tenues en 1754 au mois d'octobre qu'il y aura 30 ans au mois d'octobre prochain ${ }^{145}$ ". À cette occasion le feudiste perçoit aussi les "ventes " encore non acquittées ${ }^{146}$. Ces recettes doivent permettre de couvrir au moins les frais. Dans les comptes envoyés par Labry au prieur de Brion, dont " l'affaire est commencée depuis huit ans ", les assises de juillet 1788 ont rapporté $808110 \mathrm{~s}$ $8 \mathrm{~d}$ pour une dépense de $811 \mathrm{l} 12 \mathrm{~s}$. Aux assises tenues à Baugé en $1784^{147}$, 509 déclarations concernant onze paroisses ont été passées ${ }^{148}$, la recette totale a atteint $3026112 \mathrm{~s} 9$ d, soit, après déduction de $240 \mathrm{l} 13 \mathrm{~s} 3 \mathrm{~d}$ pour le contrôle des actes et de $245 \mathrm{l} 13 \mathrm{~s} 3$ d pour le papier, une recette de $2573 \mathrm{l}$ 14 s 6 d., dont il faut encore soustraire les frais de personnel. Les résultats attendus justifient l'organisation de la lourde opération des assises.

142. Trottier, Principes des coutumes d'Anjou et du Maine, Angers, Mame, 1783, t. 2, p. VII. 143. Fréminville considère que le seigneur " qui fait renouveler son terrier y trouve toujours des ressources par les droits [...] que les redevables cachent et recèlent autant qu'ils peuvent, dont il se fait payer " et ainsi allège le coût de l'opération; c'est pourquoi il a tout intérêt à " faire faire cette rénovation au moins tous les trente ans " (Les vrais principes..., op. cit., t. 2, p. 277).

144. Arch. dép. de Maine-et-Loire, E 612. Une " éventillation " est une description énumérative.

145. Arch. dép. de Maine-et-Loire, E 2982. Il ne s'agit pas d'instituer de nouveaux devoirs mais ceux-ci peuvent paraître tels aux paysans qui en ont perdu l'habitude.

146. Terme beaucoup plus fréquent que l'expression " lods et ventes ». Leur produit est casuel mais souvent élevé.

147. Arch. dép. de Maine-et-Loire, E 2966 ou C 254. Les chiffres fournis diffèrent mais faiblement.

148. Arch. dép. de Maine-et-Loire, E 2966. Comptes rendus par Labry à Bretonneau et Aubin. Le détail est fourni paroisse par paroisse pour les onze communautés concernées (les "dépens " sont les plus élevés à Vernantes : 728116 s 6 d; les plus faibles à Corzé, Pellouailles : 416 s et13 $13 \mathrm{~s}$ ). 


\section{Convocation et organisation}

La périodicité des assises n'est pas fixée et la tenue n'est pas régulière. L'initiative de la convocation et le choix de la date reviennent au seigneur; la prescription trentenaire lui impose de ne pas dépasser un intervalle de 29 ans.

Les sujets assignés doivent se présenter en personne et donc parfois venir de loin. Le mauvais temps fait craindre l'absentéisme et les sessions ont lieu le plus souvent entre la fin du printemps et le début de l'automne, bien que ce soit le temps des gros travaux agricoles. En décembre 1783, Bretonneau repousse les assises de Vernantes à cause de "la rigueur du froid "; en février 1784 il évoque les "gelées et neiges si opiniâtres qu'on ne sait sur quoi compter " et se dit " emprisonné par les neiges ${ }^{149}$ "; en janvier 1785 encore, il évoque « les chemins mauvais, les jours courts [qui] seraient bien incommodes aux sujets ${ }^{150}$ "; finalement les assises débutent le 20 avril. Les assises se tiennent soit chez le seigneur, dans sa demeure ou l'auditoire s'il en existe un ${ }^{151}$, soit dans un lieu clairement indiqué sur la convocation et dans lequel sont réalisés les aménagements nécessaires à l'installation des archives utiles et à la réception du public. Les assises de la châtellenie de Varains ${ }^{152}$ relevant de l'abbaye de Saint-Florent se déroulent " en la maison de la grange de Varains au bourg, paroisse dudit lieu "; en 1755 , les assises du fief de Ruzebouc, appartenant au chapitre Saint-Laud d'Angers, sont tenues " en la maison seigneuriale dud. fief ", située au village de la Pointe à Bouchemaine.

La convocation est lancée par le « bailli, juge ordinaire, civil et criminel, de police [...] sénéchal dud lieu " qui mande " au premier sergent sur ce requis d'assigner devant [lui] à la requête du procureur fiscal du fief et seigneurie de [...] les sujets et vassaux dudit fief aux assises qui se tiendront à... le... ${ }^{153}$ ". Tous sont assignés : le marquis d'Andigné, comte de Sainte-Gemmes, est convoqué aux assises tenues au château de Serrant, pour rendre foi et hommage au baron du Plessis-Macé, par ailleurs comte de Serrant, pour une de ses terres ${ }^{154}$. Pour annoncer la tenue des assises, deux voies sont employées. La convocation peut être collective, faite par

149. Arch. dép. de Maine-et-Loire, E 2978.

150. Id. Les hivers 1783 et 1784 furent particulièrement rigoureux (LEBRUN, François, Les hommes et la mort en Anjou aux $17^{e}$ et $18^{e}$ siècles, Paris/La Haye, Mouton, 1971, fig. 41).

151. La coutume de Touraine n'évoque pas expressément la tenue des assises mais impose au seigneur d'élire domicile dans le fief pour " la réception " de ses droits (Titre I, art. 6).

152. Arch. dép. de Maine-et-Loire, H 2311. Le feudiste rédige lui-même le formulaire pour les convocations et le fait imprimer (Arch. dép. de Maine-et-Loire, E 2978. Lettre de Bretonneau à Labry, décrivant les instructions données à l'imprimeur Mame pour l'impression des formulaires).

153. Arch. dép. de Maine-et-Loire, E 534. Seigneurie de l'Épinay en Combrée, le 15 octobre 1728.

154. Arch. dép. de Maine-et-Loire, E 1265. Il a évidemment recours à un procureur, Labry en l'occurrence. 
la voie ordinaire des proclamations au prône des messes paroissiales qui convoquent une assemblée générale de la communauté à laquelle les assises seront annoncées. En juin 1784, Bretonneau fait faire « les affiches et sommations pour les assemblées de Parcé et Verné ${ }^{155}$ "; en janvier 1785 il rédige pour le syndic de Linières une sommation qui « lui sera donnée mardi avec injonction d'assembler dimanche prochain ". L'absentéisme aux assemblées rend cette voie peu efficiente et le feudiste peut préférer l'affichage public. En juin 1783, Labry doit commander à l'imprimeur Mame les affiches ${ }^{156}$ pour les assises du marquisat de Jalesnes, en au moins 50 exemplaires sur papier timbré, à $3 \mathrm{~s} 10 \mathrm{~d}$ la feuille, et 25 exemplaires sur "papier non marqué ".

La convocation individuelle est plus efficace car elle touche, à son domicile, chaque individu mais elle est plus coûteuse puisqu'elle est faite par un huissier. Un délai de un à cinq jours sépare généralement la date de délivrance de l'assignation de celle de la comparution. Une liasse de convocations individuelles datées de 1778, complétées mais non distribuées, figure dans les archives de l'abbaye Saint-Florent; elles ont été préparées dans le cabinet de Labry ${ }^{157}$. Chacune est constituée d'un feuillet intitulé "Avertissement ". Au recto figure une formule imprimée que l'huissier a remplie avec la date de la convocation et celle des assises, la qualité du requérant, les nom et domicile de l'huissier et de la personne assignée "à comparaître devant mondit sieur le Sénéchal [...] Les assises tenantes [...] pour exhiber les titres en vertu desquels il est devenu propriétaire des héritages spécifiés de l'autre part, pour, après ladite exhibition, prendre par ledit procureur telles qu'il verra bon être, rendre par déclaration les héritages censifs, faire la foi et hommage, et rendre par aveu les hommages, reconnaître les services et devoirs, auxquels ils sont sujets, et répondre aux conclusions dudit procureur fiscal ${ }^{158}$ ". Une injonction rappelle : "Apportez avec vous tous les contrats d'acquêts, partages, licitations et autres actes, faits depuis trente ans; en vertu desquels vous, ou vos auteurs, êtes devenus propriétaires des héritages spécifiés de l'autre part, et de l'argent pour payer les devoirs auxquels ils sont sujets. " Le cens étant une redevance " réelle " perçue sur une terre bien identifiée alors que le détenteur peut changer, la convocation nomme en premier les lieux, et non l'individu visé. Au verso, la description des biens concernés a été copiée par un clerc d'après le papier terrier antérieur. Le censitaire peut ainsi préparer sa déclaration en comparant les titres qu'il détient, s'il en a, et cette copie, pour éventuellement contester auprès du feudiste la superficie ou la nature des biens énoncées ou même l'appartenance à la seigneurie concernée.

155. Arch. dép. de Maine-et-Loire, E 2978.

156. Il faut aussi faire imprimer les lettres à terrier, qui doivent être affichées (coût : 12 lt).

157. Arch. dép. de Maine-et-Loire, H 2314. Lettre de M. de Sourdé à Saumur, le 2 avril 1778.

158. Arch. dép. de Maine-et-Loire, H 2311. 


\section{Le feudiste aux assises}

Le feudiste n'est pas membre ès-qualité du tribunal seigneurial, même s'il est parfois écrit qu'il « tient les assises " ou qu'il " mène les assises ». La présidence revient au juge seigneurial ou sénéchal ${ }^{159}$, assisté par le procureur fiscal, un greffier, souvent par ailleurs notaire, et un sergent. Ainsi les assises de Ruzebouc sont tenues " par nous Vincent Benoist, écuyer, maire et capitaine général de la ville d'Angers, sénéchal, seul juge civil et criminel de lad. seigneurie, poursuite et diligence de $\mathrm{M}^{\mathrm{e}}$ René Charles Raimbault, seigneur de la Douve, avocat en la sénéchaussée, procureur fiscal, assisté de $\mathrm{M}^{\mathrm{e}}$ Toussaint Béraud, commis greffier ordinaire ${ }^{160}$ ". Pour certains des biens de l'abbaye Saint-Nicolas, les assises sont tenues en 1772-1776 par « René Louis Marie Martineau, conseiller du roi, docteur professeur en la faculté des droits de l'université, un des trente de l'Académie royale des sciences et belles lettres [d'Angers], sénéchal, juge ordinaire, civil et criminel et de police de la juridiction temporelle de l'abbaye ${ }^{161} "$. Toutefois les services d'un feudiste sont indispensables : "Cette tenue d'assises n'a jamais été finie, attendu que M. Labry feudiste a cessé de travailler pour les Messieurs de Saint-Nicolas en 1776 et que personne n'a depuis travaillé à ce fief " selon le cellerier ${ }^{162}$. Le feudiste assure tous les préparatifs, en particulier la mise en ordre des titres anciens pour permettre leur consultation, la convocation des sujets, puis la re-convocation des absents. Lors des audiences il est présent; témoin, il signe en tant que tel : ainsi la signature de Labry et d'Aubin figure au bas des minutes des déclarations faites à de nombreuses assises. Le feudiste peut ainsi vérifier l'exactitude, sur le fond et la forme, des déclarations, aveux et dénombrements, toute erreur pouvant être à l'origine de poursuites; en cas de " confusion dans l'ordre féodal ", il doit parvenir à débrouiller la situation. Cette tâche le retient loin d'Angers pendant de longs moments. En 1782, Labry et Aubin signent les minutes des soixante déclarations et quatre actes de foi et hommage rendus au fief de Gené entre le 15 et le 19 juillet ${ }^{163}$. En 1784, de mars à la mi-juin, puis à la fin d'août et en septembre, ils résident à Baugé pour assister aux assises ${ }^{164}$; entre ces deux périodes, en juillet ils sont à Vernantes, pour celles du marquisat de Jalesnes ${ }^{165}$. On les retrouve à partir du 16 décembre 1784 aux

159. Il semble qu'en Anjou le sénéchal de la seigneurie soit nommé sénéchal d'assises, ce qui n'est pas toujours le cas dans le Bas-Maine, où des officiers royaux comme Pichot de La Graverie ont été très souvent appelés à remplir cette fonction (ANTOINE, Annie, Fiefs et villages..., op. cit., p. 242).

160. Arch. dép. de Maine-et-Loire, G 983.

161. Arch. dép. de Maine-et-Loire, H 510.

162. Arch. dép. de Maine-et-Loire, H 510. La tenue d'assises avait débuté en 1772.

163. Arch. dép. de Maine-et-Loire, G 1248. 8 déclarations ont été reçues le 17 juillet, 11 le 18 et 8 le19.

164. Arch. dép. de Maine-et-Loire, C 254. Ils sont hébergés au château où Bretonneau leur a aménagé non sans difficultés un appartement.

165. Arch. dép. de Maine-et-Loire, 1E 387. 
assises du fief d'Avaissé, partie du comté de Sainte-Gemmes-d'Andigné ${ }^{166}$ : entre les 16 et 29 décembre, en neuf jours y sont passées 29 déclarations; ils reviennent deux jours en janvier 1785 pour cinq déclarations ${ }^{167}$. Dans tous les cas, pendant ce temps ils sont logés sur place et ne peuvent donc pas travailler sur d'autres dossiers.

\section{Fonctionnement}

Lors de leur comparution les censitaires présentent leurs titres; ils font leur reconnaissance en décrivant les biens détenus et en indiquant les rentes dues ${ }^{168}$, le tout " en laissant et baillant, à [leurs] dépens, copie collationnée à l'original " (Coutume d'Anjou, art. 4) dont ils ont assumé le coût. Ils doivent aussi payer 5 sous pour le premier article de la déclaration, $2 \mathrm{~s} 6 \mathrm{~d}$ pour les suivants, ainsi que le montant du contrôle des actes ${ }^{169}$.

Au début d'une session le rythme des comparutions est soutenu : à Varrains, entre le 17 juin et le 11 juillet 1776, le sénéchal reçoit quotidiennement (dimanche excepté) de 9 à 18 déclarations qui sont de longueur très inégale; ensuite il ralentit nettement. Les assises peuvent durer plus d'un an car elles ne fonctionnent pas en continu toute l'année et certains déclarants tardent à se présenter. Quelques-uns ne peuvent se déplacer pour venir " offrir leur obéissance " au jour dit pour diverses raisons ${ }^{170}$; toutefois ne refusant pas de comparaître, ils demandent alors un délai ou suggèrent un autre lieu, par exemple à Angers chez Labry, ou un autre interlocuteur comme un notaire ${ }^{171}$.

166. Arch. dép. de Maine-et-Loire, E 1265. Les assises sont tenues tous les jours entre le 16 et le 23 décembre (dimanche 19 excepté), puis les 27 et 29 décembre et reprennent les 10 et 11 janvier 1785 . Toutes les déclarations portent les signatures de Labry et Aubin, qui ont donc dû multiplier les déplacements. À partir de 1786, Labry ne signe plus. C'est Aubin qui a conservé la clientèle du comte de Sainte-Gemmes d'Andigné et il est sans doute l'auteur du terrier non daté, non signé, du fief et seigneurie de Sainte-Gemmes (Id., E 1257).

167. Arch. dép. de Maine-et-Loire, E 1265. En 1786, il y a encore quelques déclarations.

168. Ces documents sont une source très riche pour l'étude du paysage rural puisque les différentes natures des sols sont indiquées : terres labourables (mais sans indication sur les cultures), vignes, prés, pacages, landes, friches, bois etc.

169. BnF, F-23650 (569) [consultable sur Gallica]. Arrest du conseil du Roi du 30 avril 1718. Il affirme que la pratique des déclarations devant les assises et non devant un notaire aurait eu comme "seule vue de frustrer les droits du Contrôle " et rappelle que, même si elles ont la forme d'un acte judiciaire, les déclarations ne sont pas exemptes du contrôle des actes, dont la taxe doit être acquittée.

170. Arch. dép. de Maine-et-Loire, H 2314. Le 19 avril 1777, Aucard écrit de Saumur qu'il ne pourra pas répondre à la convocation, à cause de ses affaires et " d'une épouse que j'ai sur le point d'accoucher ". Les excuses évoquent des problèmes de santé ou liés à l'âge, des " affaires en cours ", des délais trop brefs (" une autre fois, vous me donnerez plus de temps").

171. Arch. dép. de Maine-et-Loire, E 2985. 
En cas d'absence non justifiée, un "second et dernier avertissement " est lancé; s'il reste sans effet, une assignation est envoyée ${ }^{172}$. Les récalcitrants obstinés s'exposent aux sanctions prévues par la coutume, comme la confiscation de leurs tenures. Les risques encourus ne sont pas théoriques. En décembre 1785, une sentence prononcée par le sénéchal déclare :

" au moyen de ce que lad. Lecompte, femme Trépreau, n'a comparu ni autre pour elle fondé de pouvoir, à cet effet lui en avons donné et donnons défaut et pour le profit, lecture faite dud. exploit et des titres au soutien de lad. demande, et pour les devoirs non faits et non payés, nous avons permis au procureur fiscal de faire saisir féodalement et mettre en la main du seigneur de cette cour les héritages ci-dessus confrontés; condamnons cependant lad. femme Trepreau de payer par deniers ou acquits valables vingt-neuf années d'arrérages échus aud. jour de Saint-Michel Mont Gargant (sic) dernière dud. devoir ci-dessus spécifié, aux amendes de coutume et aux dépens que nous avons liquidés à... compris le coût de l'expédition et signification des présentes ${ }^{173}$ ".

Certains sujets font preuve d'une grande force d'inertie : le 16 septembre 1774 Labry écrit de Baugé : « les B. (sic) de sujets me font pester. Depuis qu'Aubin est parti, je n'ai vu que six personnes, malgré les assignations ils ne se remuent pas plus » ou dix ans plus tard, toujours de Baugé : «[...] les sujets m'impatientent très fort; ils ne viennent point. En conséquence, pas d'argent. J'eus cependant hier lundi quelques personnes [...] mais tout cela n'est rien : sur cinq cents personnes qui doivent venir il ne m'en est venu que cent ${ }^{174}$ ". Il reste difficile de déterminer l'effectif des récalcitrants, de connaître le nombre de poursuites effectives et les effets de ces absences sur la réformation du terrier.

L'organisation et la tenue des assises demandent donc beaucoup de temps et des dépenses importantes en personnel et matériel. Elles constituent un moment fort dans la vie de la seigneurie et l'institution ne donne pas de signes d'essoufflement en 1789, comme le prouvent les archives conservées. Elles permettent au seigneur de mettre de l'ordre dans son chartrier, de faire reconnaître tous ses droits, de lutter contre les « usurpations " mais aussi de se faire payer les redevances qui lui sont dues; mais de leur côté, les vassaux et tenanciers y voient l'opportunité de se faire confirmer leurs titres de "propriété ": "les deux parties se satisfont de ce titre de propriété qui les légitime mutuellement ${ }^{175}$ ".

172. Arch. dép. de Maine-et-Loire, E 2966. Dans la seigneurie de la Roche-Noyau, dont Labry doit dresser le terrier par contrat du 15 décembre 1784, « MM les feudistes viennent de finir de tenir les assises, il y a encore quarante censitaires qui ont refusé de venir déclarer, on les fera assigner " (Id., E 2971/2). En 1784, à Baugé, le coût d'une assignation est de $3110 \mathrm{~s} 9 \mathrm{~d}$.

173. Arch. dép. de Maine-et-Loire, 1E 387.

174. Arch. dép. de Maine-et-Loire, E 2972.

175. BIANCHI, Serge, "Terriers, plans-terriers et Révolution ", dans BRUNEL, Ghislain, GuyotJEannin, Olivier et Moriceau, Jean-Marc (dir.), Terriers et plans-terriers... op. cit., p. 309-324 (ici p. 314). 
La rénovation d'un terrier est bien une entreprise de longue haleine; ainsi l'Anjou peut abriter de nombreux feudistes ${ }^{176}$ sans pour autant que l'opération ait été menée dans une majorité des seigneuries. Le nombre des seigneurs qui ont commandé la rénovation de leurs terriers ou qui au contraire ne l'ont pas fait faire, soit par désintérêt, soit par manque de moyens financiers, ne peut être connu. Il n'existe aucune preuve que des seigneurs angevins aient profité de ces rénovations pour établir de nouveaux droits même si certains ont pu remettre en vigueur des droits négligés ${ }^{177}$. À cette même époque, quelques seigneurs ont manifesté la volonté de retrouver la plénitude des pouvoirs seigneuriaux en obtenant la restauration de leur justice. Des seigneurs de vieille noblesse comme le marquis de Quatrebarbe ${ }^{178}$ et le comte de La Galissonnière, marquis de la Grande Guerche ${ }^{179}$, présentent au roi une requête, qui peut sembler anachronique, afin que soit rétablie leur justice "si nécessaire au bien public et à l'avantage des vassaux et habitants". Ils se disent mus par une volonté de bienfaisance et d'utilité mais ils expriment aussi leur fierté d'exercer le pouvoir régalien par excellence : La Galissonnière affirme « que le roi a partagé, pour ainsi dire, avec les seigneurs hauts-justiciers l'auguste partie de sa puissance, il leur a communiqué la plénitude de son pouvoir ". L'activité de ces justices n'eut évidemment qu'un temps.

Les événements du printemps 1789 n'interrompent pas l'activité des feudistes. Le travail " fait aux fiefs de Brissac " par le feudiste Gageau continue; le 24 avril 1789, Jeanne Ribault de l'Isle, dame de la Bizollière, etc., commande la rénovation du papier terrier de ses terres ${ }^{180}$. La " profession neuve $^{181}$ " de feudiste aurait-elle alors été tuée par la nuit du 4 août? Au contraire dans un premier temps, car si les décrets du 11 août abolissent le régime féodal, ils déclarent rachetables les droits " réels ". La fin de l'année 1789 a donc dû connaître un regain d'activité pour les feudistes, à la mesure de la fébrilité suscitée par ces mesures. Début octobre, les moniales de Nyoiseau pensent que tout ouvrage est devenu inutile puisque « les nou-

176. Un dénombrement reste à faire. Au contraire, dans le Bas-Maine, les feudistes sont rares mais les seigneurs semblent avoir de plus en plus recours à eux dans la seconde moitié du XVIII ${ }^{\mathrm{e}}$ siècle (ANTOINE, Annie, Fiefs et villages..., op. cit., p. 239).

177. LemarChAND, Guy, La fin du féodalisme dans le pays de Caux, Paris, CTHS, 1989, p. 295. "Le résultat de cet effort est moins la remise en vigueur de droits disparus depuis plusieurs siècles, que la perception plus rigoureuse de droits levés auparavant irrégulièrement et incomplètement. "

178. Arch. dép. de Maine-et-Loire, 1B191, fol. 5v-23v. Insinuations du greffe civil de la sénéchaussée d'Angers, 1780-1782. Le siège de la justice est fixé à Chatelain, près de Château-Gontier.

179. Arch. dép. de Maine-et-Loire, E 693. Proclamation au prône de la messe de SaintAubin-de-Luigné, le 22 décembre 1783. Cette justice a vraiment fonctionné (Id., E 694).

180. Arch. dép. de Maine-et-Loire, 285J40/34.

181. Gutton, Jean-Pierre, "Commissaires feudistes en Lyonnais et Beaujolais au XVIII ${ }^{\mathrm{e}}$ siècle ", in Populations et cultures. Études réunies en l'honneur de François Lebrun, Rennes, AFL, 1989, p. 187. 
velles ont annoncé la suppression des fiefs et des communautés ${ }^{182}$ ". Aubin leur homme d'affaires les détrompe :

"Le décret du 4 août dernier annonce bien la suppression du régime féodal mais il est dit que tous les cens, rentes, droits de mutation et autres seront rachetables, et devaient continuer à être perçus jusqu'au remboursement, dont le prix et le mode seront fixés par l'assemblée nationale. Les droits seigneuriaux casuels sont donc exigibles, jusqu'au remboursement [...] Nous n'avons point discontinué de travailler à tous les fiefs des seigneurs, ils nous ont même très fort recommandé de continuer avec toute la vigueur possible pour nous trouver en état après le règlement fait de recevoir les amortissements des frèches, ou cens $[\ldots]^{183}$."

Quand la loi du 15 mars 1790 qui organise les conditions du rachat ${ }^{184}$ stipule aussi que, désormais, "il est défendu à tout propriétaire de fiefs de continuer aucuns terriers [...] commencés avant la publication des présentes ", la profession de feudiste perd toute raison d'être. Toutefois elle ne disparaît que partiellement : elle se transforme sous l'effet de la mutation radicale du droit qui renverse l'ordre ancien et impose la propriété de type romain. Certains feudistes, dont Labry ne fit pas partie, ont en effet su mettre leur connaissance du terrain et leurs qualités professionnelles au service de nouvelles entreprises comme celle de la confection des cadastres ${ }^{185}$.

182. Arch. dép. de Maine-et-Loire, E 1506.

183. Arch. dép. de Maine-et-Loire, C 246.

184. L'article 5 du titre I précise que " les formes [...] de reconnaissances par aveux et dénombrements, déclarations à terrier [...] sont abolies " (cité par SoBoul, Albert, "De la pratique des terriers... ", loc. cit., p. 1064). La situation est ambiguë puisque pour se faire payer les seigneurs doivent pouvoir faire la preuve de leurs droits. Aubin et Letourneau, feudistes de Monsieur, écrivent à Merlin de Douai en juillet 1790 " qu'ils ont entièrement cessé leurs travaux pour les fiefs des seigneurs particuliers, que néanmoins ils se trouvent aujourd'hui forcés de parachever, étant des objets susceptibles d'être rachetés, tels que cens, rentes, lods et ventes etc. " (Id.).

185. Labry n'est pas passé au service du nouveau régime. Depuis au moins 1786 il était le feudiste du comte de Rougé, marquis de Cholet (Arch. dép. de Maine-et-Loire, E 2967); il gérait aussi sa terre de la Bellière, à Saint-Pierre-Montlimart, depuis peut-être 1787 puisque dans ses papiers se trouve un "Livre de caisse ou recettes et dépenses faites pour la Bellière " entre le 26 septembre 1787 et le 24 mars 1789 (Id., E 2971/2) et en novembre 1791, il est encore le régisseur de cette terre (Id., E 2985). Il aurait suivi l'armée vendéenne avec ses fils (PoRT, Célestin, Dictionnaire..., op. cit., t. II, p. 434). 


\section{RÉSUMÉ}

Dans plusieurs provinces, les rénovations de papiers terriers semblent se multiplier au cours des dernières décennies de l'Ancien Régime ; elles peuvent être le signe d'une réactivation du système féodal et seigneurial mais aussi la réponse à des besoins d'ordre et de clarté dans la gestion des seigneuries. Le nombre des feudistes a augmenté mais leurs effectifs restent inconnus. Les archives d'un feudiste qui a travaillé à Angers entre 1770 au moins et $\mathbf{1 7 8 9}$ permettent d'analyser ses méthodes de travail, de mesurer l'importance et la nature de sa clientèle, d'évaluer le coût de la rénovation d'un papier terrier. Cette opération passe par différentes étapes bien précises : tri et classement des titres qui constitue une tâche préliminaire indispensable, levée de plans qui bénéficie des progrès des méthodes de cartographie, recueil des déclarations des vassaux et censitaires dans le cadre, en Anjou, des assises seigneuriales, rédaction du papier terrier. Ces travaux, coûteux pour les seigneurs, demandent, même pour une petite seigneurie, beaucoup de travail de la part du feudiste, de ses clercs et de différents collaborateurs comme les géomètres ; un feudiste ne peut donc pas mener plusieurs gros chantiers en parallèle. Même à la fin de l'Ancien Régime où elle semble avoir été plus fréquente, la rénovation des terriers n'a pu concerner, sans doute, qu'une minorité de seigneuries.

\section{ABSTRACT}

In many provinces of France, renovations of papiers terriers seem to become more numerous at the end of the Ancien Régime; they may be the sign of a renewal in the feudal and manorial system as well as an answer to a need for clarity and order in the management of a lordship. Consequently, the number of feudistes, who were the specialists in seigneurial law increased but we cannot know how many were active. The papers of a feudiste who lived and worked in Angers during the last decades of the eighteenth century (before 1790) enable us to know how he worked, who and how many people asked him for the renovation of a papier terrier, how much it cost. He had to do a number of well-defined tasks. The first one was sorting and filing titles because no other work could be done without it. Maps then had to be drawn, which had become easier thanks to new cartographical methods. Finally landowners in the lordship had to notify to the seigneurial court their fiefs and censives and the taxes they had to pay for them. All this work was expensive for a lord and, even for a little lordship, and was a timeconsuming process. A feudiste, his clerks and other people such land surveyors could, therefore, not work simultaneously for a lot of lords. Even if there were more orders for renovations, only a minority of the terriers were brought up to date at the end of the Ancien Régime. 